\title{
Pattern Recognition in Pharmacodynamic Data Analysis
}

\author{
Johan Gabrielsson ${ }^{1,4}$ and Stephan Hjorth ${ }^{2,3}$
}

Received 31 August 2015; accepted 20 October 2015; published online 5 November 2015

\begin{abstract}
Pattern recognition is a key element in pharmacodynamic analyses as a first step to identify drug action and selection of a pharmacodynamic model. The essence of this process is going from data to insight through exploratory data analysis. There are few formal strategies that scientists typically use when the experiment has been done and data collected. This report attempts to ameliorate this deficit by identifying the properties of a pharmacodynamic model via dissection of the pattern revealed in responsetime data. Pattern recognition in pharmacodynamic analyses contrasts with pharmacokinetic analyses with respect to time course. Thus, the time course of drug in plasma usually differs markedly from the time course of the biomarker response, as a consequence of a myriad of interactions (transport to biophase, binding to target, activation of target and downstream mediators, physiological response, cascade and amplification of biosignals, homeostatic feedback) between the events of exposure to test compound and the occurrence of the biomarker response. Homing in on this important-but less often addressed-element, 20 datasets of varying complexity were analyzed, and from this, we summarize a set of points to consider, specifically addressing baseline behavior, number of phases in the response-time course, time delays between concentration- and response-time courses, peak shifts in response with increasing doses, saturation, and other potential nonlinearities. These strategies will hopefully give a better understanding of the complete pharmacodynamic response-time profile.
\end{abstract}

KEY WORDS: duration of response; exploratory data analysis; intensity of response; mixture dynamics; modeling; onset of action; oscillatory response; physiological limit; response half-life; response-time courses; saturation; transduction; turnover.

\section{INTRODUCTION}

During many years of project work in pharma drug research and discovery settings, we have repeatedly experienced instances where pharmacologists and kineticists/ modelers alike have utilized pharmacodynamic response data suboptimally. It is our impression that this partly resides in different terminologies and "language" used in the two disciplines, but also in differences regarding interpretation that emanates from the inherent focus on pharmacokinetics or pharmacodynamics, depending on the very inclination of the person analyzing the data. Given that important information can be lost this way, it is evident to us that an integrated view would greatly facilitate and increase power of data

"Things are much more marvelous than the scientific method allows us to conceive" (Keller 1983, pp. 198-207)

${ }^{1}$ Division of Pharmacology and Toxicology, Department of Biomedical Sciences and Veterinary Public Health, SLU, Box 7028, SE-750 07, Uppsala, Sweden.

${ }^{2}$ Department of Molecular and Clinical Medicine, Institute of Medicine, The Sahlgrenska Academy at Gothenburg University, SE-413 45, Gothenburg, Sweden.

${ }^{3}$ PharmaLot Consulting AB, V. Bäckvägen 21B, SE-434 92, Vallda, Sweden.

${ }^{4}$ To whom correspondence should be addressed. (e-mail: Johan.Gabrielsson@slu.se) analysis. In turn, this is likely to have positive repercussions on speed and cost of drug discovery and development. In this tutorial article, we endeavor to enable such integration by focusing on data patterns and identifying potential underlying factors that will further analysis and interpretation.

Pattern recognition is a key element in pharmacodynamic data analyses when first selecting a model to be regressed to data. We call this process going from data to insight exploratory data analysis. Despite being a key element toward further analysis and understanding, there are no formal best practices that scientists typically use. This report deals with identifying the properties of a pharmacodynamic model by dissecting the pattern that responsetime data reveal graphically. Pattern recognition is a pivotal activity when modeling pharmacodynamic data, because a rigorous strategy is essential for dissecting the determinants behind response-time courses. In the pharmacology field, pattern recognition has also been proposed for interpreting results of drug-drug interactions and pharmacokinetic data $(1,2)$. To inspire young kineticists beyond the slavery of computers, we have practiced pattern recognition over three decades in our pharmacology teaching. The central question is how much information one can extract from the data without falling into the trap of machine-made answers. The analyst should be in charge of the knowledge extraction prior to utilizing software.

A set of points to consider are proposed that specifically addresses exploratory data analyses, number of phases in the response-time course, convex or concave curvature, baseline 
behavior, time delays between concentration- and responsetime courses, lag time prior to drug action, peak shifts in time of the maximum response with increasing doses, saturation, shape of return to predose response, and other potential nonlinearities that are visually caught from the data. Pattern recognition in pharmacodynamic analyses also differs from pharmacokinetic analyses in that the time course of the test compound in plasma diverges markedly from the time course of the biomarker response. In pharmacokinetic pattern recognition, the agent monitored in plasma (and/or other matrices) is generally the same as the one that was originally administered.

A flowchart for the model building process is proposed in Fig. 1. Model building should ideally originate in knowledge about mechanism(s) of action, exposure, the observed consequences of several dose levels, and even repeated dosing. It is important to always put the pharmacodynamic outcome in the perspective of pharmacokinetic characteristics of the test compound; furthermore, this should always be an iterative process. Figure 1 is primarily meant as a first step to build pharmacodynamic knowledge.

\section{PRESENTATION OF CASE STUDIES}

The basic turnover model, which has been applied to several of the datasets in this tutorial, is shown in Fig. 2. This class of models is also known as the indirect response model. The production or loss processes can either be inhibited or stimulated by the drug. When stimulation occurs on the production term (turnover rate), the response increases over time, and the opposite results when there is a stimulation of the loss term (fractional turnover rate). Inhibition of production drives the response in the opposite direction (decline), whereas inhibition of the loss term increases the response. This basic model can then be extended to a myriad of permutations to capture the drug mechanisms and responsetime patterns.

Figure 3 shows a schematic overview of the 20 data schemes to be discussed in this paper. These correspond to patterns typically encountered in in vivo pharmacodynamic practice and which to a great extent can be characterized by means of turnover models $(3,4){ }^{1}$ In some cases, alternative models (other permutations of the proposed turnover model or link models or binding on/off models) may be more appropriate. The diagram in Fig. 1 was therefore compiled to briefly highlight alternatives. We have intentionally focused on turnover concepts given their unparalleled ability to schematically mimic and explain pharmacodynamic responses. For each case study, we will provide an underlying model including the differential equations that describes the pattern(s) (system), parameters, constants, and number of functions involved in the regression analysis. Underlying

\footnotetext{
${ }^{1}$ Some of the datasets are generously shared by different companies under confidentiality terms; hence, their therapeutic origin or mechanism of action is not revealed. Needless to say, ideally, information about the study designs, pharmacological mechanism(s), and/or true variability in the original data would have improved the analysis. However, even in the absence of such detail, the presented datasets still serve the purpose of pattern recognition. In some cases, alternative models (other permutations of the proposed turnover model or link models or binding on/off models) may be more appropriate.
}

biological contributory factors will likewise be discussed in the same context.

- Case studies 1-4 illustrate the basic four turnover (indirect response) models with inhibition on production (case study 1) or inhibition on loss (case study 2), and stimulation of production (case study 3 ) or stimulation on loss (case study 4). These four examples are covered more in detail with respect to plasma kinetics, drug mechanism(s), and pharmacodynamics. See also Jusko and Ko for additional drug examples matching these four cases (5).

- Case studies 5-8 represent models with gradually increasing complexity. The lack of baseline and display of a linear (saturable) decline seen in case study 5 can be compared to the basic turnover model in case study 3. Case study 6 involves inhibition and stimulation of $k_{\text {in }}$ arising from dual actions of an agent. See Paalzow and Edlund for additional drug examples of this pattern (6). A concentrationresponse plot is also supplied due to its interesting pattern. A synergistic system of simultaneous stimulation of $k_{\text {in }}$ and inhibition of $k_{\text {out }}$ is shown in case study 7 . Case study 8 shows a system of delayed onset of action, saturable intensity, peak shifts, and a monotonic return toward the baseline and is therefore distinctly different from case study 3 .

- Case studies 9-11 demonstrate irreversible drug actions, exemplified by cell kill (case study 9), bacterial kill (case study 10), and enzyme removal (case study 11) datasets. Case study 10 displays an upper physiological limit. See also Jumbe et al. and Zhi et al. for other examples of tumor volume models and bacterial kill, respectively $(7,8)$.

- Case studies 12 and 13 illustrate pattern recognition from a dose-response-time data perspective. Case study 12 contains antinociceptive data from iv and sc dosing with a delayed onset of action, peak shifts with increasing doses, saturation, and model-dependent decline toward the baseline. Case study 13 shows a model that could be used when handling biological factors such as transduction and downstream events explaining the delayed response initiation and decline pattern.

- Case studies 14-15 show oscillating baselines resulting from the presence of an endogenous ligand (agonist; case study 14) and a time-dependent turnover rate $k_{\text {in }}(t)$ (case study 15), respectively. Case study 15 emphasizes the utility of simultaneously fitting several sources of data. See also Chakraborty et al. for modeling oscillating baseline concentrations of cortisol (9).

- Case studies 16-20 all illustrate various kinds of adaptation ranging from the gene to the functional receptor response level. Case study 16 demonstrates a single-dose profile to an antilipolytic agent, including tolerance as well as rebound. Case studies 18 and 19 exhibit clear rebound patterns, whereas in case studies 17 and 20, response approaches the baseline monotonically due to slow plasma kinetics $\left(t_{1 / 2}\right.$ plasma $>t_{1 / 2}$ response). Case studies 17-20 are 


\section{Exploration of exposure/response data}

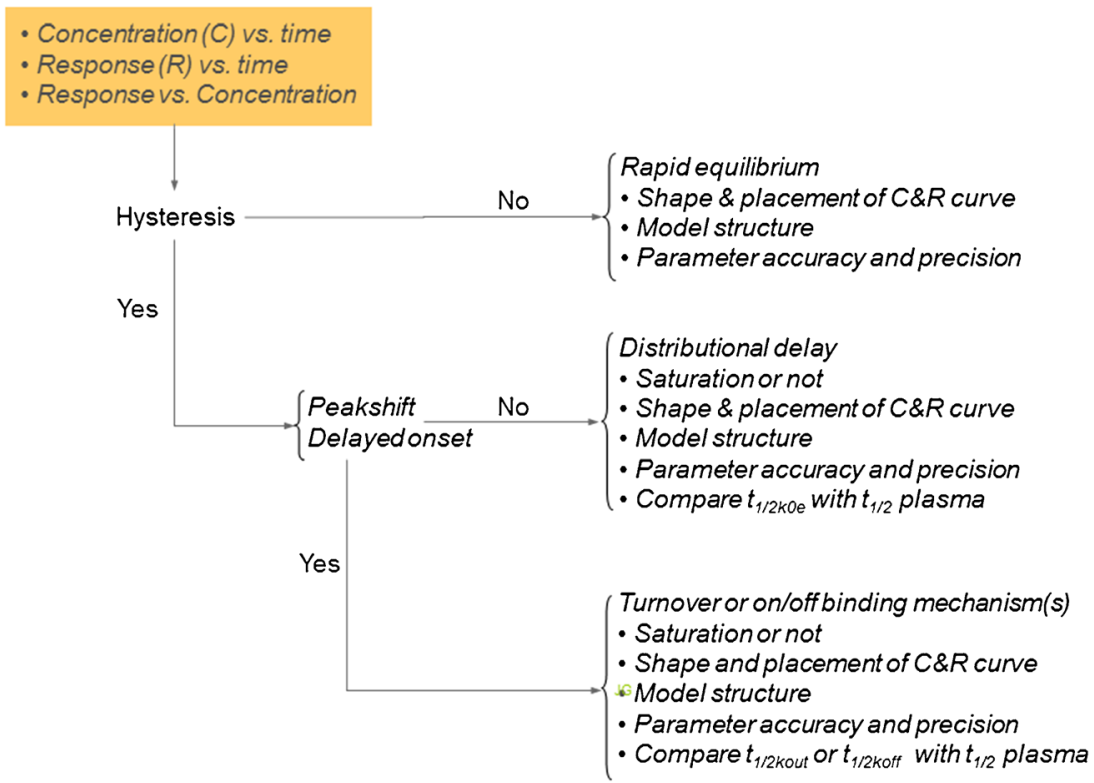

Fig. 1. Schematic diagram of some points to consider toward model setup. Start by plotting concentration- and response-time data and combine the two into a concentration-response plot, yielding hysteresis if there are time delays (diagnostic plots). If there is no obvious hysteresis (rapid equilibrium), the shape and placement of data along the concentration axis reveal the model structure. Apply an instantaneous (direct) response model to either response-time data or concentration-response data. If time delays are obvious in the diagnostic plots, then consider either a link, turnover or binding on/off model. Check for delayed onset of action and peak shifts in the $R$ - $t$ courses with increasing doses. If peak shifts exist, try either a turnover model or a binding on/off model. Let parameter accuracy and precision be part of the model selection process. Compare half-life of response $\left(t_{1 / 2 \mathrm{ke} 0}\right.$, $\left.t_{1 / 2 \mathrm{kout}}, t_{1 / 2 \mathrm{koff}}\right)$ with half-life of drug in plasma for rate-limiting step. Remember that mechanistic information should, whenever possible, drive the model building process. Fit all available response-time data simultaneously

modeled by means of a feedback model demonstrating its capacity to capture complex response-time courses. A concentration-response plot is also supplied for case study 18 due to its intriguing pattern. Ramakrishnan et al. present an extended feedback mechanism-based pharmacodynamic model (10).

A schematic picture of the turnover models is shown in Fig. 4.

The four basic turnover (indirect response) models for a diverse range of pharmacological mechanisms and response-

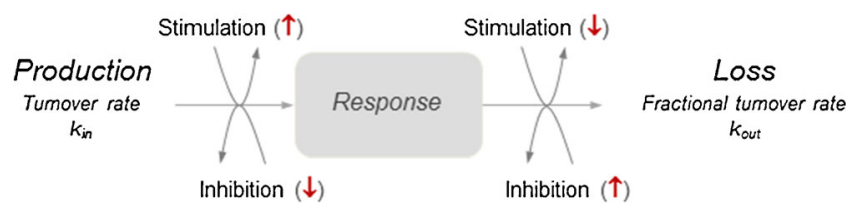

Fig. 2. Principal components of the basic turnover model, also known as the indirect response model, applied for case studies 1-4. Inhibition of $k_{\text {in }}$ causes a response $R$ to decline. Stimulation of $k_{\text {in }}$ results in an increased response. Inhibition of $k_{\text {out }}$ gives increased response and stimulation of $k_{\text {out }}$ a decreased response. Red arrows indicate the direction of the response relative to its baseline when a drug is either stimulating or inhibiting (3) time patterns have been reviewed previously (5). It was concluded that turnover models are relevant in pharmacokinetic and pharmacodynamic modeling for which the production or loss of a biomarker is responsible for the action of drugs. Our report focuses on the dissection of causes of the shapes that make up the response-time course, thereby increasing our understanding of the complete response-time profile. In order to make typical features emerge more clearly, we have therefore collected and adapted a set of patterns extracted from literature data.

The number of parameters which can be calculated for a given model is not as easily derived by a function as can be done for the majority of pharmacokinetic models $(2,11)$. One of the reasons for this is that in pharmacokinetic models, the dose is known as well as how it is administered, and the concentration-time course of the same compound is measured in plasma. By comparison, in pharmacodynamics, a drug is administered and measured in, e.g., plasma, but the response monitored is a consequence not only of drug exposure (and dose) per se but also a plethora of other interactions (cascade of events, feedback/adaptation/compensation mechanisms, synergy, saturation, transduction, endogenous agonists, other experimental conditions including environmental aspects, etc.). In that sense, pharmacokinetic processes are more predictable (often first-order absorption and disposition 

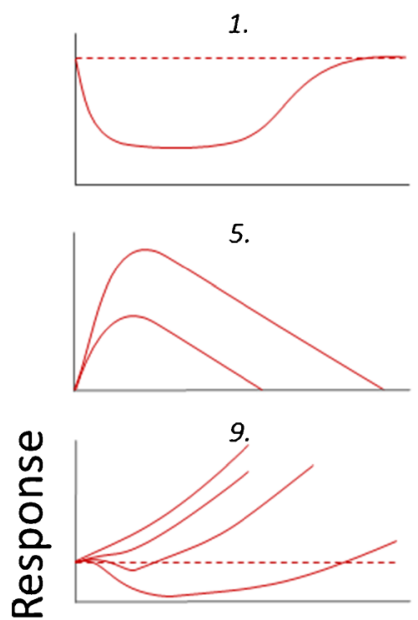

13.
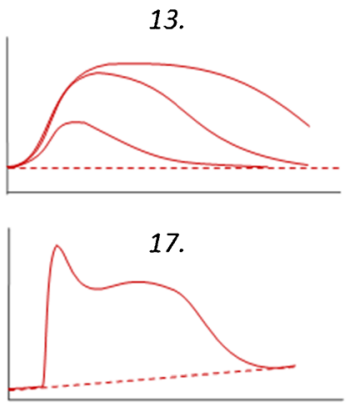

2.

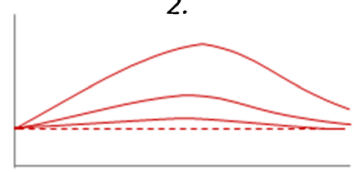

6.

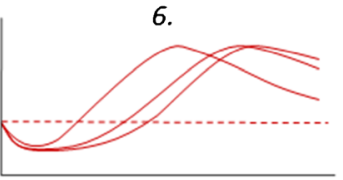

10.

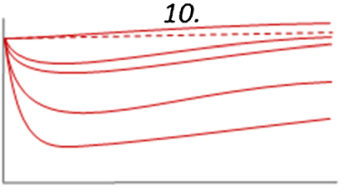

14.
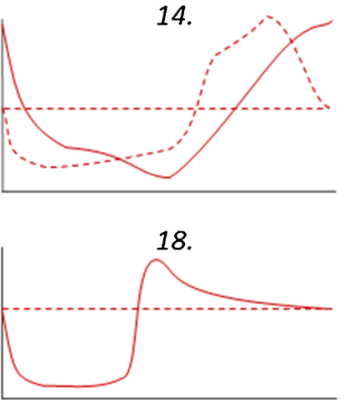

3.
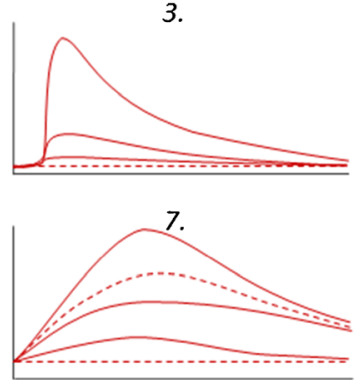

11.

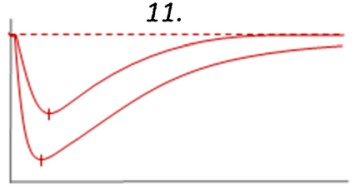

15.

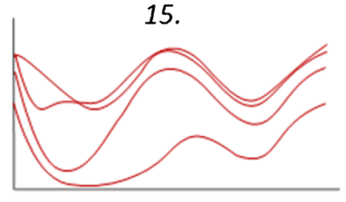

19.

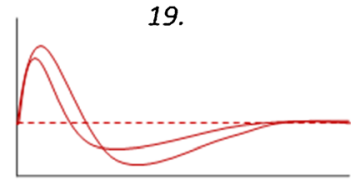

4.

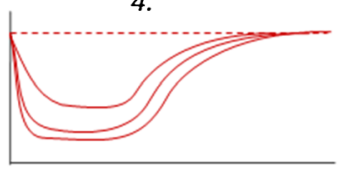

8.

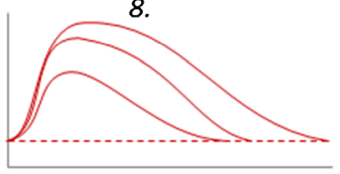

12.
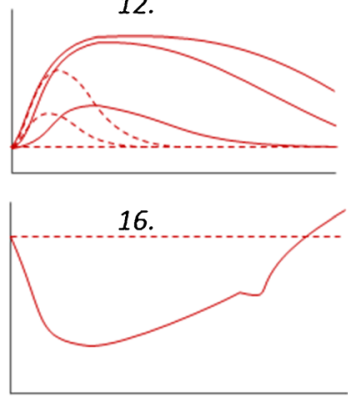

20.

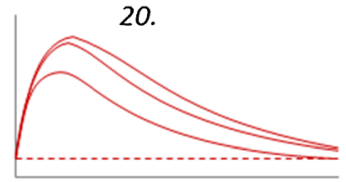

Time

Fig. 3. Schematic illustration of the 20 data patterns (4) discussed in this report with the baseline response shown by the horizontal dashed line in all case studies except number 5, where the baseline equals zero. 1 . Inhibition of production of response. 2. Inhibition of loss of response. 3. Stimulation of production of response. 4. Stimulation of loss of response. 5. Biomarker response (locomotor activity) starting from a baseline essentially equal to zero. 6 . Multiple site simultaneous action. 7. Synergistic action. 8. Transduction modeling. 9. Cell growth/kill model. 10. Bacterial cell growth/kill modeling. 11. Irreversible enzyme inhibition. 12. Dose-response-time data analysis-multiple routes of administration. 13. Doseresponse-time data analysis-transduction. 14. Oscillatory hormone model driven by endogenous agonist exposure. 15. Oscillatory baseline driven by a time-dependent turnover rate. 16. Pool/precursor model. 17. Negative feedback and drifting baseline. 18. Negative feedback using a push-and-pull model. 19. Gene regulation model. 20. Negative feedback modeling

kinetics, sums of exponentials, rarely nonlinear at pharmacological concentrations) compared to the pharmacodynamic response. The latter can seldom or never be approximated by a sum of exponentials, does not follow the principle of superposition, is often highly nonlinear, may assume any variety of atypical baseline behavior, may display circadian/ circannual or other types of rhythm(s), and is frequently a mixture of endogenous agonist and drug(s) effects and subject to control or modulation by homeostatic feedback mechanisms.

We assume that the underlying plasma kinetics are known in the majority of case studies and can be represented by a sum of exponentials. The nonlinear behavior shown in the response-time courses is therefore a consequence of nonlinear pharmacodynamic processes and is not confounded by the kinetics. In case studies 5,13 , and 18, we apply a doseresponse-time data analysis and estimate the biophase parameters simultaneously with the pharmacodynamic parameters, since no data describing exposure to drug are available.

A set of points to consider are proposed that may help guide the model selection unless the mechanism of action is known.

\section{Case Study 1}

In this study, the kinetic/dynamic relationship was studied for compound $\mathrm{X}$, intended to inhibit enzymatic production of a protein (amyloid precursor protein, APP) in the CNS, believed to impact Alzheimer's disease. Data were pooled from a group of mice which had received a single dose of $100 \mu \mathrm{g} \mathrm{kg}^{-1}$ orally of compound X (Fig. 5, upper graph (4)). The brain concentration of one of the fragments resulting from APP cleavage $\left(A \beta_{1-40}\right)$ was used as pharmacodynamic response biomarker. Subgroups of two to three animals were sacrificed at different time points to generate plasma concentration- and response (enzyme activity $=\mathrm{A} \beta_{1-40}$ formation levels)-time courses. Vehicle/controls showed a constant enzyme baseline activity over time. Plasma concentrations peaked at $30 \mathrm{~min}$, while the minimum enzyme activity in the brain (maximal pharmacodynamic response) occurred at 2$2.5 \mathrm{~h}$, thus demonstrating a clear time delay of about $2 \mathrm{~h}$ between plasma concentration and enzyme activity. The enzyme activity remained stably suppressed for $2 \mathrm{~h}$ after drug treatment before a $\sim 4$-h recovery time to baseline ensued. Based on the findings, the exposure $v s$. response data analysis aimed at fitting a turnover model with inhibitory action of production of 


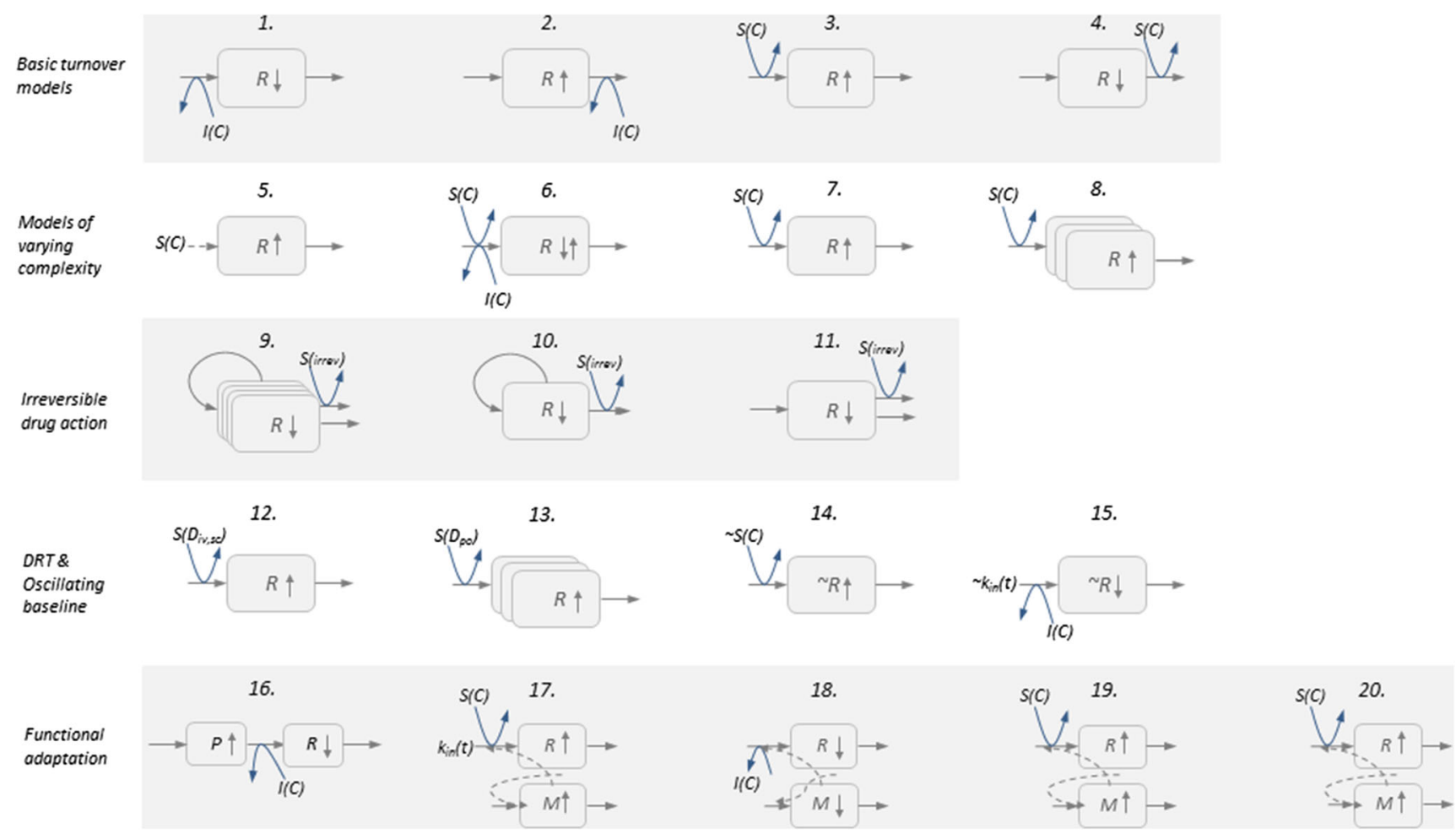

Fig. 4. Turnover models used for each of the 20 case studies schematically shown in Fig. 3 (4). $R$ and $M$ are the response and moderator compartments, respectively. The turnover rate $k_{\text {in }}$ (arbitrary unit $\mathrm{t}^{-1}$ ) is the production (synthesis, secretion) of response and the fractional turnover rate $k_{\text {out }}$ is the loss; $k_{\text {in }}$ is typically a zero-order process and $k_{\text {out }}$ first-order $\left(\mathrm{t}^{-1}\right) . I(C), S(C), S(D)$, and $S($ irrev) denote the inhibitory and stimulatory drug mechanism functions driven by plasma concentration, the stimulatory function driven by the biophase amount in doseresponse-time $(D R T)$ data analyses, and the irreversible drug mechanism function used for tumor kill, bacterial kill, and irreversible enzyme binding, respectively. $\sim S(C)$ denotes the oscillatory drug stimulation. $S\left(A_{\mathrm{b} \text { iv,po }}\right)$ and $S\left(A_{\mathrm{b}}\right.$ po $)$ are the stimulatory drug mechanism functions driven by biophase amount after iv/po and po administration, respectively. Case studies 1-20 will demonstrate different permutations of various levels of complexity

response (i.e., leading to downstream suppression of APP fragmentation) and simulate the equilibrium concentrationresponse relationship with the final parameter estimates from the regression.

When data are plotted in time order, the concentrationresponse relationship shows a clear disequilibrium, illustrated by a clockwise hysteresis curve (Fig. 5, bottom left graph). Note also the saturation of response which occurs over the 5to $25-\mu \mathrm{M}$ concentration range. The latter graph emphasizes the nonlinear inhibitory nature of the exposure-response relationship which reaches saturation at about $5 \mu \mathrm{M}$.

As seen in Fig. 5, there is an initial 30-min period with simultaneous increases in plasma concentration and enzyme suppression. However, thereafter, enzyme activity displays an apparent independent time course relative to test compound plasma exposure. From the biological perspective, such a pattern might suggest that the enzyme inhibition has a rapid onset (due to short half-life) but is also irreversible, and thus resynthesis of new enzyme molecules (in the absence of test compound exposure) is required for reinstatement of full function.

The apparently independent time course of suppressed enzyme activity shown in Fig. 5 (bottom right graph) in relation to test compound plasma levels can be expressed mathematically as a first-order input/output (Eq. 1) with inhibitory drug action (Eq. 2) on the production of response (direct inhibition of the turnover rate of enzyme activity).
Equation 1 represents the joint impact of drug absorption and elimination processes.

$C=A \cdot\left(e^{-K \cdot t}-e^{-K_{a} \cdot t}\right)=32.1 \cdot\left(e^{-0.605 \cdot t}-e^{-8.8 \cdot t}\right)$

The kinetic parameters $\left(A, K\right.$, and $K_{\mathrm{a}}$, representing a macro constant conglomerate of dose and other model parameters, elimination rate constant, and absorption rate constant, respectively) were fixed to their estimated values. This function then served to drive the nonlinear drug "mechanism" function $I(C)$, Eq. 2, when fitting the turnover model (Eq. 3) to response-time data. The plasma concentration-time curve only serves as a smooth to "drive" the inhibitory drug "mechanism" function (Eq. 2).

$I(C)=1-\frac{I_{\max } \cdot C^{n}}{\mathrm{IC}_{50}+C^{n}}$

$I(C)$ means that the inhibitory action is a function of the plasma drug concentration $C . I(C)$ acts directly on factors responsible for production of response, namely the turnover rate $k_{\text {in. }} I_{\text {max }}, \mathrm{IC}_{50}$, and $n$ denote the test compound efficacy parameter, the potency, and the sigmoidicity factor, respectively. 

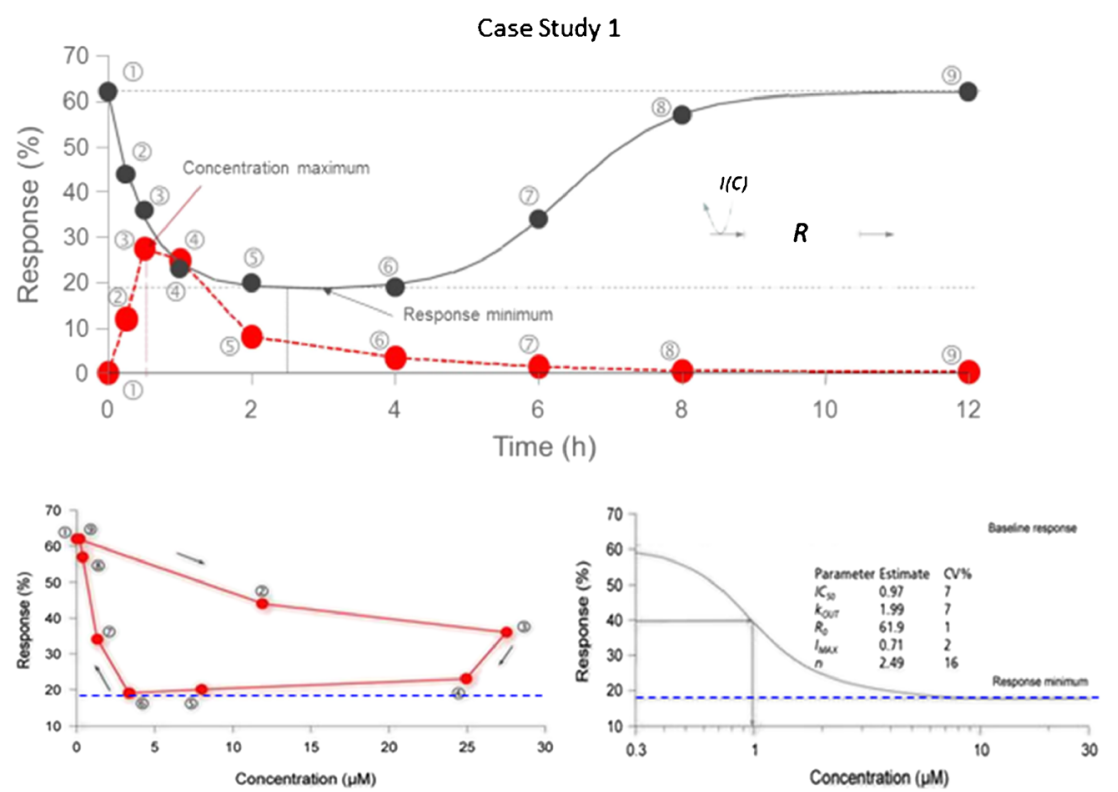

Fig. 5. Upper graph: plot of concentration-time and response (enzyme activity)-time data in mice after an oral dose of $100 \mu \mathrm{g} \mathrm{kg}^{-1}$. A clear time delay is seen between the two time courses. A flat 2-h time course is seen in the response in spite of declining plasma concentrations of the test compound. Bottom left: observed concentration-response data plotted in time order. Note the clockwise hysteresis shown by the gray arrows and the 5 to $25 \mu \mathrm{M}$ concentration interval where the (enzyme inhibitory) response appears saturated. Bottom right: semilogarithmic plot of the predicted concentration-response relationship of compound $\mathrm{X}$. The final parameter estimates of the system and drug parameters together with their individual precisions $(\mathrm{CV} \%)$ are also included. Note that this equilibrium function (Eq. 3, bottom row) lacks the hysteresis loop when plotted since time is no longer an issue. The blue dashed line in the two bottom graphs depicts the minimum response level

The turnover of response can be described mathematically by Eq. 3 below:

$$
\left\{\begin{array}{l}
\frac{\mathrm{d} R}{\mathrm{~d} t}=\text { production } \cdot \text { inhibition }- \text { loss } \\
\text { Baseline }=R_{0}=\frac{\text { production }}{\text { loss }}=\frac{k_{\text {in }}}{k_{\mathrm{out}}} \\
\frac{\mathrm{d} R}{\mathrm{~d} t}=k_{\mathrm{in}} \cdot I(C)-k_{\mathrm{out}} \cdot R \\
R_{\mathrm{ss}}=\frac{k_{\mathrm{in}}}{k_{\mathrm{out}}} \cdot I(C)=R_{0} \cdot\left(1-\frac{I_{\mathrm{max}} \cdot C_{\mathrm{ss}}^{n}}{\mathrm{IC}_{50}^{n}+C_{\mathrm{ss}}^{n}}\right)
\end{array}\right.
$$

The basic turnover model contains two parameters, the turnover rate $k_{\text {in }}$ and the fractional turnover rate $k_{\text {out }}$. The baseline value is the ratio of $k_{\text {in }}$ to $k_{\text {out }}$. The turnover rate, also called production in Eq. 3, is directly inhibited by the drug "mechanism" function $I(C)$ (Eq. 2). The drug is not acting directly on the response $R$ (A $\beta_{1-40}$ concentration) per se but inhibits directly one of the factors $k_{\text {in }}$ that governs the level of response $R$. Another classic example of this type of drug intervention is the inhibitory action of warfarin on vitamin $\mathrm{K}$ which is responsible for the production of prothrombin complex activity (12). By inhibiting the regeneration of vitamin $\mathrm{K}$ by means of warfarin, the prothrombin complex activity decreases, leading to impaired coagulation.
By fitting a mechanism-based pharmacodynamic model (Eq. 3) to the response-time data (in Fig. 5, upper graph), we obtain estimates of both systems parameters $\left(k_{\text {in }}\right.$ and $\left.k_{\text {out }}\right)$ and drug parameters $\left(I_{\max }, \mathrm{IC}_{50}\right)$. Simulating the equilibrium relationship between concentration and response in Eq. 3 (bottom line) with the final parameter estimates yields the equilibrium concentration-response relationship in Fig. 5 (bottom right graph).

Note that enzyme activity response starts at the baseline value $R_{0}$ and then gradually decreases down toward a lower minimum value $R_{\min }$ as the concentration of inhibitor $C$ increases (Fig. 5, bottom right graph).

The key features of the patterns in this case study are

- A pharmacodynamic biomarker that starts at a stable baseline and that is suppressed over time after drug treatment

- A time delay between the concentration peak and response trough

- A saturated response which displays as a flat portion of the response-time course

A plot of the concentration-response data in time order reveals a clear counterclockwise response and a sustained response over a wide concentration range. The mechanism of action is via direct nonlinear inhibition of the turnover rate of response. The time delay is captured by a turnover model, representing production of new enzyme molecules in the absence of drug exposure, and subsequent functional 
restoration. This allows us to fit a mechanism-based turnover model combined with a two-parameter nonlinear drug "mechanism" function to the data. The equilibrium concentrationresponse relationship is then simulated using Eq. 4 and the final parameter estimates from the regression of response-time data.

\section{Case Study 2}

The test compound was administered as a 6-h constant rate infusion at three dose levels. A pharmacodynamic biomarker was simultaneously measured over time (Fig. 6, case study 2 (3)). The mechanism of drug action in this case is by inhibition of the loss of response. A comparable, classic, example of such pharmacological action is the inhibitory action of furosemide on the NKCC symporter-mediated reuptake of water from urine back to blood, thereby resulting in an increased urinary excretion rate $(5,15)$.

The baseline response in the data ranges between 40 and 45 units. Dose-normalized areas of the response-time course above baseline indicated saturation when plotted against dose administered, which then suggests a saturable (nonlinear) drug "mechanism" function. A slight peak shift in the response is seen with increasing doses of the drug. The pharmacological response still rises for about $1 \mathrm{~h}$ after stopping the constant rate of infusion at $6 \mathrm{~h}$ at the highest dose level. This also suggests that maximal drug action (saturation) remains for a while in spite of declining plasma exposure to the test compound. When plasma concentrations are plotted against response, an atypical disequilibrium appears as counterclockwise hysteresis curves. The concentration maximum appears earlier than the response maximum.

The drug has kinetic properties of mono-exponential disposition with volume of distribution $V$ of $40 \mathrm{~L}$ and elimination rate constant $K 0.9 \mathrm{~h}^{-1}$. Equation 4 was first fit to the plasma kinetics.

\section{Case Study 2}

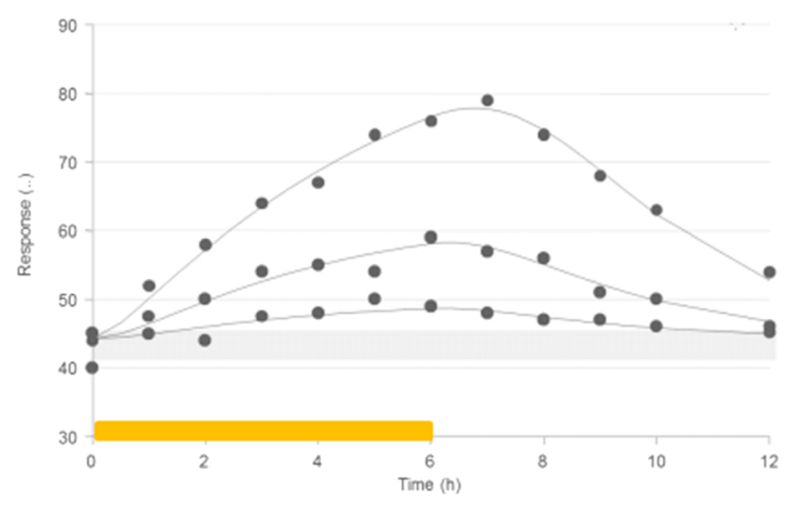

Case Study 4

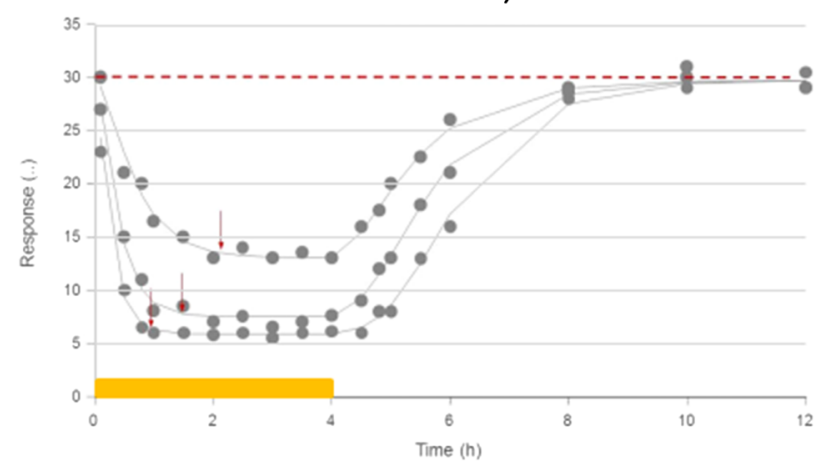

$\frac{\mathrm{d} C}{\mathrm{~d} t}=\frac{\mathrm{In}}{V}-K \cdot C$

Fig. 6. Case study 2: observed (filled symbols) and model predicted (solid lines, Eq. 7) response-time data following three 6-h constant rate intravenous infusion levels. The gray horizontal bar at 40-45 response units represents the baseline variability. The yellow bar shows the length of infusion (4). Case study 3: observed (filled symbols) and model predicted (lines, Eq. 9) response-time data at three oral dose levels of 10.75, 43 , and $172 \mathrm{mg} \mathrm{kg}^{-1}$ given at $45 \mathrm{~min}$, respectively, to rats. Note that the responses peak at approximately the same time after each dose (4). Case study 4: observed (filled symbols) and model predicted (lines, Eq. 13) response-time data after three constant rate intravenous infusions of compound $\mathrm{X}$ to patients during $4 \mathrm{~h}$ at $6400,32,000$, and 160,000 dose units. The horizontal red dashed line indicates the baseline value at about 30 units. The drug acts via stimulation of the fractional turnover rate $k_{\text {out }}$. The vertical red arrows show the time to pharmacodynamic steady state. Note that the time to steady state and the onset of action are shortened with increasing doses. The yellow bar shows the length of infusion (4). Case study 5: locomotor activity scores (counts per minute)-time data following two ip 3.12 and $5.62 \mu \mathrm{g} \mathrm{kg}^{-1}$ doses of dexamphetamine $(13,14)$. Note the apparently linear and parallel decline in response over time independent of dose. The dashed and solid lines are the resulting model fits when using a bolus- or a first-order input/output biophase model, respectively 
In is the infusion regimen. It is also known that the drug intervenes with factors controlling the loss of response (Eqs. 5 and 6).

$I(C)=1-\frac{I_{\max } \cdot C}{\mathrm{IC}_{50}+C}$

Similar to, for example, case studies $4,8,12$, and 13 , dose-normalized areas-under-the-response-time curves $\mathrm{AUC}_{R}$ decreased when plotted against the dose. This suggests a nonlinear drug "mechanism" function. Inhibition of the loss of response in Eq. 6 is then substituted by $I(C)$.

$$
\left\{\begin{array}{l}
\frac{\mathrm{d} R}{\mathrm{~d} t}=\text { production-loss } \cdot \text { inhibition } \\
\frac{\mathrm{d} R}{\mathrm{~d} t}=k_{\mathrm{in}}-k_{\mathrm{out}} \cdot I(C) \cdot R \\
R_{0}=\frac{\text { production }}{\text { loss }}=\frac{k_{\mathrm{in}}}{k_{\mathrm{out}}} \\
R_{\mathrm{ss}}=\frac{k_{\mathrm{in}}}{k_{\mathrm{out}}} \cdot \frac{1}{I(C)}=R_{0} \cdot \frac{1}{I(C)}
\end{array}\right.
$$

In Fig. 6 (upper left graph), the time courses of response are shown at three dose (exposure) levels. Note that pharmacodynamic steady state is not reached within the 6-h infusion for any of the three infusion regimens. Thus, the initial slopes are not the true values of $k_{\text {in }}$ due to the incomplete inhibitory effect of the drug on the fractional turnover rate. However, a good approximation of $k_{\text {in }}$ is obtained from the initial rising slope at the highest response-time course.

The key patterns in response-time and concentrationresponse data are

- The nondrifting baseline (obtained from a separate study)

- The stimulatory (increase in) response-time courses

- Saturation obtained from dose-normalized areas under the response-time courses above baseline

- Peak shifts with increasing exposure levels

- The relatively rapid asymptotic return to baseline upon cessation of infusion

All these features collectively suggest that a turnover model with inhibitory action of the loss of response is a good starting point, which is also the mechanism of action.

\section{Case Study 3}

Three oral doses of a new compound, acting on the urinary bladder sphincter muscle via stimulatory alpha2receptor action, were given to three groups of rats, and a physiological biomarker (voiding volume) was measured frequently over an 8-h period (Fig. 6, case study 3 (4)). Data show a predose baseline value $\left(R_{0}\right)$, a rapid onset of action with maximum response within $1 \mathrm{~h}$ after dosing, a lack of saturation with increasing doses, and a dose-dependent duration of response above the baseline.

A simple one-compartment model could describe the plasma kinetics of the drug within the studied dose range. The kinetic parameters served as input to the stimulatory function of the response model. The volume of distribution $V$ and elimination rate constant $K$ were $5.2 \mathrm{~L}$ and $0.46 \mathrm{~h}^{-1}$, respectively.

An exponential stimulatory drug "mechanism" function is proposed, which includes a concentration parameter $\mathrm{EC}_{50}$ corresponding to the plasma concentration where the response is twice the baseline. This structure is proposed since there is no tendency toward observed saturation with increasing doses. The stimulatory drug "mechanism" function is written as

$S(C)=1+\frac{C_{\mathrm{ss}}^{n}}{\mathrm{EC}_{50}^{n}}=1+\left(\frac{C_{\mathrm{ss}}}{\mathrm{EC}_{50}}\right)^{n}$

where the exponent $n$ can be set as a parameter or fixed to a constant value of 1 to avoid overparameterization of the model. Equation 8 represents the drug response originating from direct stimulation of the production of the response (i.e., stimulation of buildup with lack of peak shift, Fig. 6, upper right)

$$
\left\{\begin{array}{l}
\frac{\mathrm{d} R}{\mathrm{~d} t}=\text { production } \cdot \text { stimulation }- \text { loss } \\
\text { Baseline }=R_{0}=\frac{\text { production }}{\text { loss }}=\frac{k_{\text {in }}}{k_{\text {out }}} \\
\frac{\mathrm{d} R}{\mathrm{~d} t}=k_{\mathrm{in}} \cdot S(C)-k_{\mathrm{out}} \cdot R \\
R_{\mathrm{ss}}=\frac{k_{\mathrm{in}}}{k_{\mathrm{out}}} \cdot S(C)=R_{0} \cdot S(C)=R_{0} \cdot\left(1+\left(\frac{C_{\mathrm{ss}}}{\mathrm{EC}_{50}}\right)\right)
\end{array}\right.
$$

The time to pharmacodynamic steady state is governed by the half-life of $k_{\text {out }}$ assuming a constant plasma concentration $C_{\mathrm{ss}}$. The level of response at steady state $\left(R_{\mathrm{ss}}\right)$ is independent of time but dependent on the actual plasma drug concentration $C_{\mathrm{ss}}$ (and on baseline response $R_{0}$ ) so that

$$
R_{\mathrm{ss}}=R_{0} \cdot\left(1+\left(\frac{\mathrm{EC}_{50}}{\mathrm{EC}_{50}}\right)^{n}\right)=R_{0} \cdot 2
$$

The value of parameterizing the pharmacodynamic model this way (Eq. 7) becomes evident when $C_{\mathrm{ss}}$ equals the $\mathrm{EC}_{50}$ value and the response is increased $100 \%$ from its baseline value (Eq. 9, where $R_{\mathrm{ss}}$ becomes 2 times the baseline value $R_{0}$ ).

The key pattern seen in experimental data are

- A nondrifting baseline response (obtained from a separate study)

- A time delay between peak concentration and peak response

- A dose-dependent rise in response, with no peak shift in response with higher doses

- A lack of saturation (information obtained from dosenormalized area under the response-time curves above the baseline) at higher doses

- An asymptotic return toward the predose baseline value

This pattern is captured by a turnover model with linear (exponent $n=1$ ) stimulatory action on the production of 
response. Another example of a biological system principally comparable from the pattern point of view is the stimulatory action of erythropoietin on red blood cell count (16).

\section{Case Study 4}

This case study demonstrates the response-inhibitory action of a test compound given as a constant infusion during $4 \mathrm{~h}$ at three rates. The response starts at a defined baseline and is suppressed by the test compound (Fig. 6, case study 4 (4)). The onset of action is dose-dependent in that an increase in exposure to test compound shortens the onset of action and the time to pharmacodynamic steady state. The mechanism of action is via stimulation of factors responsible for the loss of response $\left(S(C) \cdot k_{\text {out }}\right)$. The response exhibits its nadir at the highest exposure to test compound and very little added effect is seen relative to the intermediate-dose level in spite of a 5-fold increase in dose between the intermediate- and highdose groups. There is also a 1-h delay before return toward baseline with the highest dose group, and no rebound is observed upon reaching predrug baseline response level again. Dose-normalized areas of the response-time courses also display nonlinearities when plotted against dose, suggesting that $S(C)$ is a saturable nonlinear expression. For less than $1 \mathrm{~h}$, the three time courses back to baseline rise in parallel. Another example where increased drug exposure may lead to a similar time-response pattern is the observed body weight suppression after $\mathrm{CB}_{1}$-receptor antagonists. Such agents are anorexigenic but will also at higher exposure increase energy expenditure and thereby increase the burning of body fat, both processes in turn contributing to body weight loss (17).

Equation 10 was first fit to the plasma kinetics.

$\frac{\mathrm{d} C}{\mathrm{~d} t}=\frac{\mathrm{In}}{V}-K \cdot C$

In represents the 4 -h infusion regimens. The test compound acts on the loss of response (Fig. 6, bottom left graph) according to a nonlinear (saturable) drug "mechanism" function, where $S_{\max }, \mathrm{SC}_{50}$, and $n$ are the drug efficacy parameter, potency, and sigmoidicity factor (exponent), respectively.

$S(C)=1+\frac{S_{\mathrm{max}} \cdot C_{\mathrm{ss}}^{n}}{\mathrm{SC}_{50}^{n}+C_{\mathrm{ss}}^{n}}$

which then enters the function of turnover of response (Eq. 12)

$$
\left\{\begin{array}{l}
\frac{\mathrm{d} R}{\mathrm{~d} t}=\text { production }- \text { loss } \cdot \text { stimulation } \\
\text { Baseline }=\frac{\text { production }}{\text { loss }}=\frac{k_{\mathrm{in}}}{k_{\mathrm{out}}} \\
\frac{\mathrm{d} R}{\mathrm{~d} t}=k_{\mathrm{in}}-k_{\mathrm{out}} \cdot R \cdot S(C) \\
R_{\mathrm{ss}}=\frac{k_{\mathrm{in}}}{k_{\mathrm{out}}} \cdot \frac{1}{S(C)}=R_{0} \cdot \frac{1}{1+\frac{S_{\mathrm{max}} \cdot C_{\mathrm{ss}}^{n}}{\mathrm{SC}_{50}^{n}+C_{\mathrm{ss}}^{n}}}
\end{array}\right.
$$
maximum drug-induced efficacy, potency, and the Hill parameterized with $S_{\max }, \mathrm{SD}_{50}$, and $n$ corresponding to the exponent, respectively (14). Note that the drug "mechanism" function (Eq. 14) lacks the constant term $(1+\ldots)$ typically found in these functions since no baseline information is

Note that pharmacodynamic steady state is reached at about $2 \mathrm{~h}$ for the low infusion regimen and at less than $1 \mathrm{~h}$ for the high infusion regimen (Fig. 6, bottom left graph).

The key patterns obtained from visual inspection of response-time and concentration-response data are

- A nondrifting baseline (obtained from a separate study)

- A concentration (dose)-dependent suppression of response

- Time delays between concentration and response (hysteresis)

- A faster onset of action and shorter time to pharmacodynamic steady state with increasing exposure to test compound

- A saturation of response (as judged from dosenormalized areas under the response-time courses)

- An asymptotic return toward baseline

A turnover model with saturable stimulatory action on the loss of response adequately captured the observed responsetime courses. The pattern, particularly the shortened time to onset seen at increased exposure, is consistent with a drug mechanism involving enhanced loss of the pharmacodynamic response. This might be due to actions at a single process, or-in case the pharmacodynamic response is controlled by multiple systems, like body weight-the recruitment of two or more processes that are influenced by the same target (as exemplified in the discussion of $C B_{1}$ antagonists above (17).

Data were digitized from van Rossum and van Koppen on locomotor activity score after intraperitoneal administration of dexamphetamine to rats at two dose levels (Fig. 6, case study $5(13,15))$. Data suit the purpose of dose-response-time modeling since the resolution is high, with adequate granularity in both the rise and decline in response and a clear peak shift with dose, and two dose levels were used. We will show that the slope of the post-peak linear decline in the locomotor activity score was independent of dose (3.12 and $\left.5.62 \mu \mathrm{g} \mathrm{kg}^{-1}\right)$.

A biophase model was fitted mimicking a first-order input into the biophase compartment (Eq. 13), resulting in the functions where $A_{\mathrm{b}}$ denotes the amount in the biophase

$A_{\mathrm{b}}(t)=D \cdot K^{\prime} \cdot t \cdot e^{-K^{\prime} \cdot t}$

The stimulatory drug "mechanism" function is

$\left\{\begin{array}{l}S\left(A_{\mathrm{b}}\right)=\frac{S_{\max } \cdot A_{\mathrm{b}}^{n}}{\mathrm{SD}_{50}^{n}+A_{\mathrm{b}}^{n}} \\ S\left(A_{\mathrm{b}}(0)\right)=0\end{array}\right.$

\section{Case Study 5}


available. This means that in the absence of drug, there will be no buildup of locomotor activity, which may perhaps seem a little odd. However, the group reporting these data has in other similar studies allowed 1 or more hours of habituation to experimental cages before recording the motor activity data. Such preadaptation minimizes the exploratory behavior otherwise expressed by rodents put in a novel environment, particularly if carried out during the light phase of the 24-h cycle, thus explaining the very low level of locomotor activity before and after the period of drug challenge. Modeling turnover rate $k_{\text {in }}$ and the $S_{\max }$ expression did not improve the fit but resulted in practically unidentifiable parameter values.

The drug "mechanism" function is then incorporated into the systems equation of the pharmacological response

$$
\left\{\begin{array}{l}
\frac{\mathrm{d} R}{\mathrm{~d} t}=S\left(A_{\mathrm{b}}\right)-k_{\text {out }}(R) \cdot R \\
k_{\text {out }}(R)=k_{\text {out } \max } \cdot \frac{1}{k_{\mathrm{M}}+R} \\
R_{\mathrm{ss}}\left(A_{\mathrm{b}}\right)=k_{\mathrm{M}} \cdot \frac{S\left(A_{\mathrm{b}}\right)}{k_{\text {out, max }}-S\left(A_{\mathrm{b}}\right)}
\end{array}\right.
$$

where $k_{\text {in }}$ has been removed since the model lacks a baseline value of locomotor activity. The $k_{\text {out }}(R)$ term is the response-dependent fractional turnover rate constant (Eq. 15, middle row). In light of the linear decay of response (Fig. 6), the loss of response will be modeled as a saturable term $k_{\text {out }}(R)$ with a fractional turnover rate $k_{\text {out }}$ as a function of $R$.

The pharmacological response will reach an equilibrium state if the rate of production is less than the maximal rate of loss, i.e., when the first term in Eq. 15 (upper row) is smaller than the maximal upper bound of the second term, i.e., if $S\left(A_{\mathrm{b}}\right)<k_{\text {out }}$. The equilibrium biophase amount-response relationship is given by Eq. 15 (bottom row).

The data are rich since it contains high-resolution response-time at two dose levels. It appears that the dexamphetamine-locomotor activity scores is a linear relation of the time post-peak independent of dose. Equation 15 (middle row) is therefore suggested as a reasonable approximation of the zero-order decline of response-time data. The model is simultaneously fit to both response-time courses. Dose-normalized areas increased with dose which indicates some kind of saturation in the loss of response and/or saturable stimulation function which is also supported by the peak shift in the response-time courses.

The pattern of case study 5 deviates from that of case study 3 in that the former lacks a baseline and has a linear post-peak decline in contrast to case study 3 which has a curve-linear decline.

The key patterns of this dataset are

- A near-zero baseline

- A rapid rise in the onset of action

- A dose-dependent peak shift and linear post-peak response decline

The peak shift indicates nonlinear stimulation and the dose-independent parallel post-peak decline suggests saturable (nonlinear fractional turnover rate) elimination. The near-zero baseline (or nonobservable baseline) is dealt with by removing the turnover rate from the equations. Remember that the analysis of response-time data is generally improved by access to the actual exposure profile(s) driving the response.

\section{Case Study 6}

Data were collected from a clinical study of the new Alzheimer compound, $\gamma$-secretase (GSECR) inhibitor LY450139 (18). Oral doses (40, 100, $140 \mathrm{mg}$ ) gave a dosedependent reduction in the plasma $A \beta_{1-40}$ concentrations which then returned toward baseline in a dose-dependent manner (Fig. 7, case study 4). All doses gave a substantial rebound effect, suggested to be due to the release of peripheral depots of stored $A \beta_{1-40}$. The suppression of $A \beta_{1-}$ ${ }_{40}$ lasted for 6,9 , and $11 \mathrm{~h}$ in the low-, intermediate-, and highdose groups. The integral of the unwanted rebound effects was substantially larger than the integral of suppressed response.

As referred to above, low concentrations of GSECR inhibitors may have a stimulatory effect on the $\gamma$-secretase complexes expressed in the peripheral but not central tissue(s). This stimulatory effect in peripheral tissues is overcome by inhibition at higher concentrations (Siemers et al. (18)). The proposed mechanism-based turnover model may thus be expressed
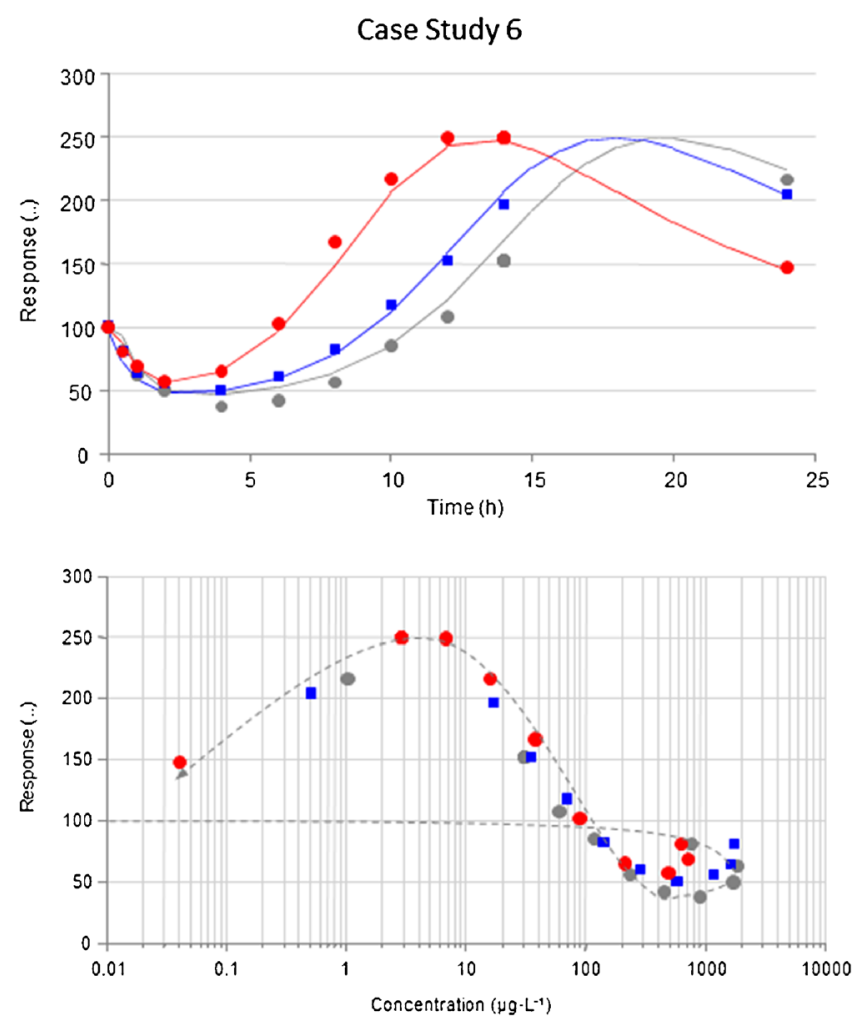

Fig. 7. Upper graph: observed (filled symbols) and model predicted (lines) response-time data in plasma after oral administration of LY450139 to human volunteers. Data scanned from Siemers et al. (18). Dataset analyzed with a dual action (inhibitory/stimulatory) on the turnover rate of response (Eq. 16). Bottom graph: concentrationresponse plot with data from all three dose groups collated 


$$
\left\{\begin{aligned}
\frac{\mathrm{d} R}{\mathrm{~d} t} & =\text { production } \cdot \text { inhibition } \cdot \text { stimulation }- \text { loss } \\
R_{0} & =\frac{k_{\mathrm{in}}}{k_{\mathrm{out}}} \\
\frac{\mathrm{d} R}{\mathrm{~d} t} & =k_{\mathrm{in}} \cdot I(C) \cdot S(C)-k_{\mathrm{out}} \cdot R \\
R_{\mathrm{ss}} & =\frac{k_{\mathrm{in}}}{k_{\mathrm{out}}} \cdot I(C) \cdot S(C)
\end{aligned}\right.
$$

There is a pronounced inhibition (reduction) of response at high concentrations where $I(C)$ will dominate over $S(C)$ ( $\mathrm{IC}_{50}$ still high in comparison to $\mathrm{SC}_{50}$ ). At low concentrations, the $S(C)$ term dominates and a response above baseline $R_{0}$ is established (Eq. 16, bottom row). Alternative models have been presented elsewhere (19).

The key patterns of this dataset are

- A defined predose baseline value

- An initial dose-dependent suppression followed by

- A dramatic rebound of response, which displays

- A peak shift with increasing doses

This biphasic behavior is modeled by means of dual action inhibitory/stimulatory drug mechanism functions. Suppression of response occurs at high initial plasma concentrations of the test compound (inhibitory action) and rebound which is a consequence of a predominant stimulatory action restricted to a peripheral vs. central compartment at lower concentrations.

\section{Case Study 7}

The CNS activity of compounds A and B have been established in a series of preclinical studies, and a recent analysis pointed at some important aspects of synergistic effects during concomitant treatment with $\mathrm{A}$ and $\mathrm{B}$ in certain acute psychotic disorders (Fig. 8, case study 7 (4)). Compound A inhibits biochemically the removal of a CNS mediator, while compound B stimulates the release of the same mediator, both mechanisms resulting in an increased synaptic presence of the mediator. Combined exposure to A and $\mathrm{B}$ shortens the time of onset of response, increases the intensity beyond prediction, and extends the duration of the response. The baseline has been shown to be stable within the observational time frame. It is evident that inhibiting the removal of the mediator $(=I(C)$ in the schematic) by compound A while at the same time enhancing the output $(=S(C)$ in the schematic) by compound $\mathrm{B}$ will result in a marked accentuation of the final response. Moreover, a steeper slope of biomarker response buildup is seen after compound A compared to compound B (Fig. 7, case study 7). This indicates that, at the exposures in the example, slowing the rate of mediator removal has a stronger impact on the response than does the facilitation of release. Thus, while based on the combined mechanisms of $\mathrm{A}$ and $\mathrm{B}$, a synergistic response might certainly be predicted, the strong augmentation of response attained upon their combination disclosed further insights on the underlying biology of the processes and their relative importance in this context. In turn, such knowledge might also help to further understand the pathophysiology of the condition to be treated, and potentially adjusting component dosing protocols to optimize therapy. $^{2}$

The exposure profiles of compounds $\mathrm{A}$ and $\mathrm{B}$ were modeled separately and then served as input to $S\left(C_{\mathrm{B}}\right)$ and $I\left(C_{\mathrm{A}}\right)$, respectively. The turnover model of the CNS responsetime data is shown in Fig. 4 (case study 7) together with the proposed mechanisms of action; stimulation of the release (= $\left.S\left(C_{\mathrm{B}}\right) \cdot k_{\mathrm{in}}\right)$ and inhibition of removal $\left(=I\left(C_{\mathrm{A}}\right) \cdot k_{\mathrm{out}} \cdot R\right)$ of the mediator. The turnover of response $\mathrm{d} R / \mathrm{d} t$ can be described by Eq. 17

$$
\left\{\begin{array}{l}
\frac{\mathrm{d} R}{\mathrm{~d} t}=\text { production } \cdot \text { stimulation-loss } \cdot \text { inhibition } \\
\frac{\mathrm{d} R}{\mathrm{~d} t}=k_{\mathrm{in}} \cdot S\left(C_{\mathrm{B}}\right)-k_{\mathrm{out}} \cdot I\left(C_{\mathrm{A}}\right) \cdot R \\
R_{\mathrm{ss}}=R_{0} \cdot \frac{S\left(C_{\mathrm{B}}\right)}{I\left(C_{\mathrm{A}}\right)}=R_{0} \cdot \frac{1+\frac{E_{\mathrm{max}} \cdot C_{\mathrm{B}}}{\mathrm{EC}_{50, \mathrm{~B}}+C_{\mathrm{B}}}}{1-\frac{I_{\max } \cdot C_{\mathrm{A}}^{n}}{\mathrm{IC}_{50, \mathrm{~A}}^{n}+C_{\mathrm{A}}^{n}}}
\end{array}\right.
$$

where $k_{\text {in }}$ is the turnover rate (production), $k_{\text {out }}$ the fractional turnover rate (loss), $S\left(C_{\mathrm{B}}\right)$ the stimulatory action of compound $\mathrm{B}$, and $I\left(C_{\mathrm{A}}\right)$ the inhibitory action of compound $\mathrm{A}$. Equation 17 can be rearranged for prediction of the steadystate response $R_{\mathrm{ss}}$ under continuous exposure to $\mathrm{A}$ and $\mathrm{B}$. Equation 17 (bottom) also states that the combined impact of the two test compounds is synergistic $S\left(C_{\mathrm{B}}\right) / I\left(C_{\mathrm{A}}\right)$ rather than additive $S\left(C_{\mathrm{B}}\right)+I\left(C_{\mathrm{A}}\right)$, where $E_{\max }, \mathrm{EC}_{50 \mathrm{~B}}, I_{\max }$, and $\mathrm{IC}_{50 \mathrm{~A}}$ are the efficacy and potency of compounds $\mathrm{B}$ and $\mathrm{A}$, respectively. Prior information supports the conclusion that $I_{\max }$ can be set to unity (i.e., there is total blockade of loss of response at a high enough exposure to compound $\mathrm{A}$ ).

The key patterns of this dataset are

- A stable baseline (obtained from a separate experiment)

- A slow rise in response by either stimulation of $k_{\text {in }}$ or inhibition of $k_{\text {out }}$

- Synergistic action when compounds are given simultaneously

This pattern is elucidated by administration of two compounds alone or combined. The onset of action is slow, reaches a peak, and then declines back to the baseline value. When given together, a faster onset of action, a higher intensity of response, and a longer duration of action are seen. This greater than additive (i.e., synergistic) action when compounds are combined can be explained by their cooperative effect originating from simultaneous stimulation of production of response and inhibition of its loss. Also, a faster return of the response back to baseline with the combination treatment may be indicative either of rapid tolerance development or the early cessation of inhibitory action by agent $A$ on factors determining the loss of response (20).

\footnotetext{
2 An "additive" drug "mechanism" on the response by two compounds results in a $1+1=2$ response. A "multiplicative" (synergistic) drug "mechanism" on the response results in a greater response than the sum of the individual contributions.
} 
Case Study 7

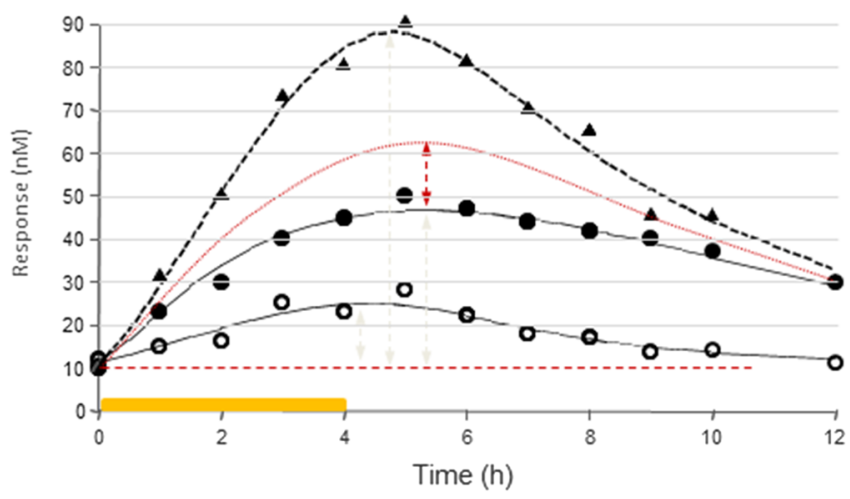

Case Study 9

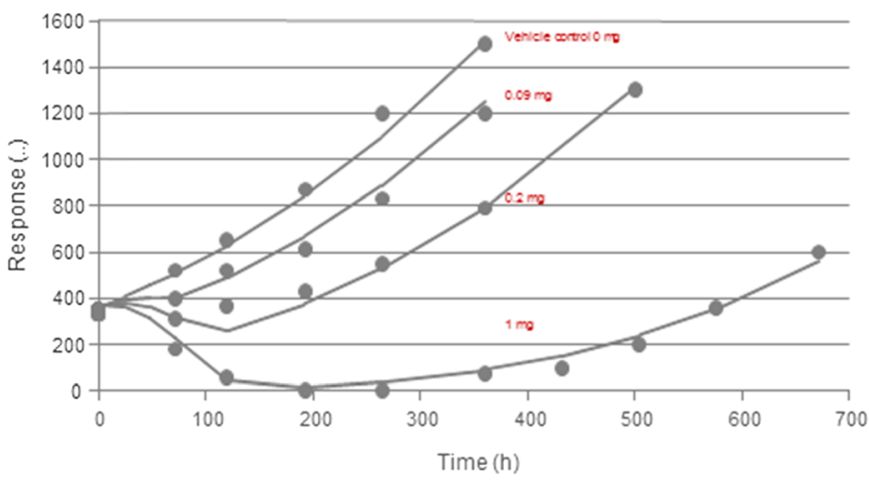

Case Study 8

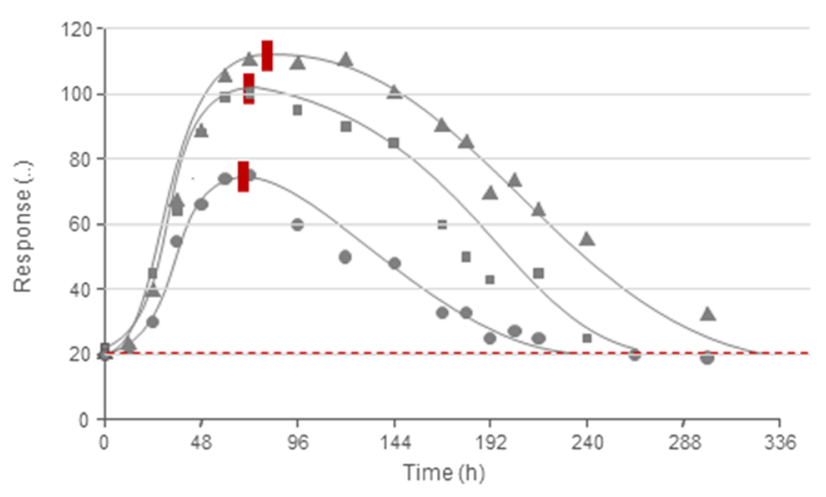

Case Study 10

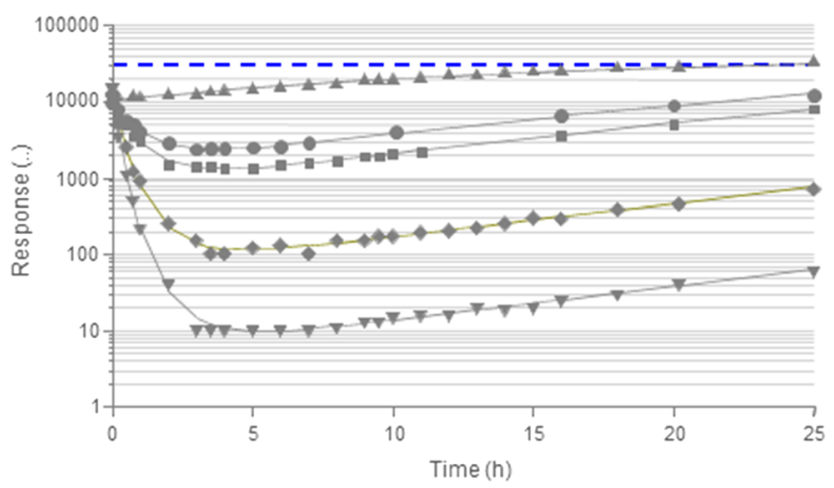

Fig. 8. Case study 7: observed (filled circles) and model predicted (solid lines) response-time data of the stimulatory effect of compound B on production of response (filled circles; middle time course), inhibitory effect of compound A on loss of response (open circles; bottom time course), and the combined response of the two after simultaneous dosing (filled triangles; top time course). Compounds A and B were infused over a period of $4 \mathrm{~h}$ with a total amount of 4000 and $8000 \mu \mathrm{mol}$, respectively. The red curve and bidirectional arrows show the theoretically predicted outcome of a strictly additive drug action of compounds A and B relative to compound B alone. The yellow bar shows the length of infusion (4). Case study 8: observed (symbols) and predicted (lines) response-time data. Note the concave rise in response during the first $\sim 36 \mathrm{~h}$ after dosing. The maximum response displays a tendency toward saturation at the highest dose. There is also a small peak shift with increasing doses, which is due to the nonlinear stimulatory function (Eq. 20). The dashed horizontal line shows the constant baseline value (4). Case study 9: linear plot of observed (solid symbols) and model predicted (lines) tumor volume-time data in a number of mice. The vehicle control (0 mg) displays an exponential growth in tumor volume, whereas the other dose groups show a dose-dependent time shift in the growth/regrowth curves. The two highest dose groups also exhibit a stalled tumor growth for $\sim 200 \mathrm{~h}$ and shrinkage in tumor volume for $\sim 600 \mathrm{~h}$, respectively (4). Case study 10: observed (symbols) and predicted (lines) response (bacterial count) vs. time after 1, 2, 4, and $8 \mu \mathrm{g}$ of a new antibiotic $U$-FU. Note the large range of response counts (5 orders of magnitude) (4)

\section{Case Study 8}

Concentration-time data and response-time readouts in this case study were obtained from an anonymized set of human data. The study design had the following characteristics: stable baseline during the run-in period (Fig. 8, case study 8 (4)), three dose levels in an ascending dose protocol fashion, and tested in the same subject at three separate occasions. A 20-24-h delay was observed for the onset of biomarker response which increased in a dose-dependent fashion. A peak shift was observed with increasing doses, manifesting as a tendency toward saturation at the two highest doses. Dose-normalized areas under the responsetime courses declined when plotted against dose, strengthening the notion that the stimulatory response is saturable. Intensity of response increased less than dose-proportionately. The biomarker response waned toward the baseline, after a relatively constant value for $72 \mathrm{~h}$ at the highest dose.
A mono-exponential model was fit to the concentration-time data. The nonlinear drug stimulatory function is written as

$$
S(C)=1+\frac{E_{\max } \cdot C}{\mathrm{EC}_{50}+C}
$$

The turnover model for interaction between ligand $C$ and receptor $R_{\text {rec }}$ is

$$
\begin{aligned}
\frac{\mathrm{d} R_{\text {rec }}}{\mathrm{d} t} & =k_{\text {in }} \cdot S(C)-k_{\text {out }} \cdot R_{\text {rec }} \\
& =k_{\text {out }} \cdot\left(R_{0} \cdot S(C)-R_{\text {rec }}\right)=\frac{1}{\tau} \cdot\left(R_{0} \cdot S(C)-R_{\text {rec }}\right)
\end{aligned}
$$

where $\tau$ is the transit time through each intermediate compartment. That is, $\tau$ corresponds to the time needed to 
transit and convey the signal via a chain of mediators further and further downstream from the initial point of drug-target interaction. The turnover of response in each transit compartment in the chain from receptor $R_{\text {rec }}$ (Eq. 19) to observed response $R_{4}$ becomes

$$
\left\{\begin{array}{l}
\frac{\mathrm{d} R_{1}}{\mathrm{~d} t}=\frac{1}{\tau} \cdot\left(R_{\mathrm{rec}}-R_{1}\right)=k_{\mathrm{out}} \cdot\left(R_{\mathrm{rec}}-R_{1}\right) \\
\frac{\mathrm{d} R_{2}}{\mathrm{~d} t}=\frac{1}{\tau} \cdot\left(R_{1}-R_{2}\right)=k_{\mathrm{out}} \cdot\left(R_{1}-R_{2}\right) \\
\frac{\mathrm{d} R_{3}}{\mathrm{~d} t}=\frac{1}{\tau} \cdot\left(R_{2}-R_{3}\right)=k_{\text {out }} \cdot\left(R_{2}-R_{3}\right) \\
\frac{\mathrm{d} R_{4}}{\mathrm{~d} t}=\frac{1}{\tau} \cdot\left(R_{3}-R_{4}\right)=k_{\text {out }} \cdot\left(R_{3}-R_{4}\right)
\end{array}\right.
$$

where $R_{4}$ is the function for the observed functional response. Note that the model can be parameterized either with $k_{\text {out }}$ or the transit time $\tau$, where $k_{\text {out }}$ equals $1 / \tau$.

The key patterns of this dataset are

- A stable baseline (obtained from a separate experiment)

- A delayed onset of action that may be mimicked by one or more "transit" compartments

- Peak shifts in response with increasing doses

- Saturation at the highest dose

- A less than dose-proportional increase in response

- A slow return of the response toward the baseline

The 20-24-h delay in the onset of action along with the aforementioned list of pattern characteristics suggests that the biomarker response observed is located downstream of the initial drug-target mechanism. A series of transit compartments was thus introduced in the model to capture the slow onset of action, and a nonlinear drug mechanism function used to tailor saturable peak response at the highest dose. A constant baseline value was assumed for the observed time range.

\section{Case Study 9}

This case study highlights modeling of tissue growth/kill by means of a first-order growth second-order kill model. Data were collected on tumor xenograft volume after vehicle control and a single dose of $0.5,2$, and $10 \mu \mathrm{mol} \mathrm{kg}{ }^{-1}$ of a test compound given orally to mice (Fig. 8, case study 9 (4)). The tumor volume data displayed an exponential increase over time in the vehicle/control group $(0 \mathrm{mg})$, thus an upwardly drifting baseline reflecting the balance between tissue growth and death in the absence of drug treatment. The tumor volume response was shifted to the right with increasing doses, and in the highest dose group (1 mg), there was also a temporary decrease lasting $\sim 600 \mathrm{~h}$, until the tumor size eventually returned toward predose baseline. Tumor regrowth occurred in a near-parallel fashion to the control group with the two lower dose regimens, but appeared somewhat slower with the highest dose (at least for the duration of the experiment). These observations might suggest that the lower doses primarily act on the tumor growth process, whereas the highest dose additionally incorporates an effect on the "kill" mechanisms.

The basic turnover of the natural cell growth and cell death is given by Eq. 21 (upper row). It is however assumed that few cells are actually killed and removed in the vehicle control group within the studied time frame, therefore resulting in the approximation represented by the simplified expression shown in the bottom row of Eq. 21.

$$
\left\{\begin{array}{l}
\frac{\mathrm{d} R}{\mathrm{~d} t}=k_{\text {growth }} \cdot R^{2 / 3}-k_{\mathrm{kill}} \cdot R^{2 / 3} \\
\frac{\mathrm{d} R}{\mathrm{~d} t}=k_{\text {growth }} \cdot R^{2 / 3}
\end{array}\right.
$$

In the presence of the test compound, turnover of cell growth and cell kill becomes

$\left\{\begin{array}{l}\frac{\mathrm{d} R_{1}}{\mathrm{~d} t}=k_{\text {growth }} \cdot R_{1}^{2 / 3}-k_{\text {kill }} \cdot R_{1}^{2 / 3} \cdot S(C) \\ \frac{\mathrm{d} R_{2}}{\mathrm{~d} t}=k_{\text {kill }} \cdot R_{1}^{2 / 3} \cdot S(C)-k_{\text {kill }} \cdot R_{2}^{2 / 3} \\ \frac{\mathrm{d} R_{3}}{\mathrm{~d} t}=k_{\mathrm{kill}} \cdot R_{2}^{2 / 3}-k_{\mathrm{kill}} \cdot R_{3}^{2 / 3} \\ \frac{\mathrm{d} R_{4}}{\mathrm{~d} t}=k_{\mathrm{kill}} \cdot R_{3}^{2 / 3}-k_{\mathrm{kill}} \cdot R_{4}^{2 / 3}\end{array}\right.$

The $2 / 3$ power term was suggested by Jumbe et al. (7), to account for a spherically based tumor shape and growth. While it may be applicable in other contexts also, it should not be used by default. The cytotoxic action of the test compound $S(C)$ is given as a nonlinear function of test compound concentration, the maximum kill rate capacity, and a parameter $\mathrm{KC}_{50}$ which corresponds to the plasma test compound concentration at which the kill rate has reached $50 \%$ of maximal capacity.

$S(C)=k_{\mathrm{kill}, \max } \cdot \frac{C^{n}}{\mathrm{KC}_{50}^{n}+C^{n}}$

which is a saturable kill process where the exponent $n$ is fixed to 1 . The $k_{\text {kill,max }}$ parameter is unitless and is simply a scaling factor of $k_{\text {kill }}$. The model assumes that when no drug is onboard, there will be no killing process, only tumor growth. To avoid this limitation of the original model, we suggest that Eq. 24 is rewritten as

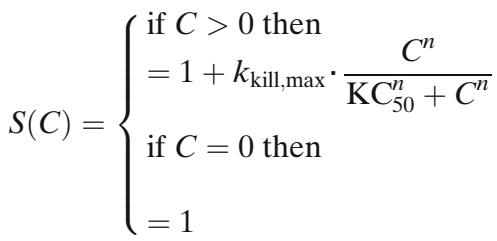

This means that $S(C)$ is either the full expression (Eq. 24, upper row) when drug is present or 1 (Eq. 24, bottom row) when no drug is present.

$\mathrm{TV}(t)$ represents the tumor volume measured at time $t$, where the initial $(t=0)$ tumor volume is $\operatorname{TV}(0)$,

$\mathrm{TV}(t)=V\left(R_{1}\right)+\ldots . .+V\left(R_{n}\right)$ 
The number of compartments of cell proliferation and death is very much dependent on the resolution of experimental data and the duration of the initial plateau in tumor volume upon dosing of the test compound. A total of four compartments for the tumor volume $\mathrm{TV}(t)$ is used in this example which could be tested by adding or removing a transit compartment at a time to the model. The number of transit compartments that generates the lowest objective function value is typically chosen.

The tumor static concentration TSC of a single test compound system, whereby tumor growth and death rates are equal and tumor volume remains unchanged, is derived below:

$\mathrm{TSC}=\frac{k_{\text {growth }} \cdot \mathrm{KC}_{50}}{k_{\text {kill }} \cdot k_{\text {kill,max }}-k_{\text {growth }}}$

The calculated TSC provides insight on drug efficacy and the in vivo sensitivity of the tumor cells (7). This concentration also provides guidance for optimal dose selection when developed for drug combinations.

The key patterns of this dataset are

- A drifting baseline, displaying as exponential growth in the control group

- A dose-dependent increase in the duration of drug action before tumor growth recurs

- A possible differential impact of lower vs. high dose on cell growth and kill processes

The growth of tumor volume vs. time displays an exponential growth in the vehicle control group and then a dose-dependent rightward time shift in tumor volume growth with increasing drug doses. The intermediate dose seems to stall growth for $\sim 200 \mathrm{~h}$, whereas the high-dose group showed shrinkage in tumor volume below the initial value for $\sim 600 \mathrm{~h}$. Data are lacking an upper physiological limit of tumor volume which may have repercussions on the actual parameter estimates. Disclaimer: Data in tumor volume studies are often truncated in the volume or time dimension due to ethical constraints. This may result either in errors in the model structure and/or in biased parameter estimates. The use of switches between the exponential and linear phases in growth rates is biologically implausible and should therefore be avoided whenever a mechanistic interpretation of models and parameters is sought.

\section{Case Study 10}

In this example, a new potent antibacterial compound was being developed. To establish its potency in a resistant bacterial strain, a 10,000 unit dose of bacteria was injected into the bloodstream of four groups of Wistar rats (Fig. 8, case study 10 (4)). Doses of 1, 2, 4, or $8 \mu \mathrm{g} \mathrm{kg}$-1 of the antibiotic were given to each of the groups, respectively. The bacterial count in the vehicle/ control $(0 \mu \mathrm{g})$ group rose by approximately 3 -fold within the 25-h time frame of the experiment. The drug dose groups displayed a dose-dependent lowering in bacterial count and a shortening in onset of action with increasing dose. All curves show parallel first-order bacterial regrowth over time. The upper "physiological" limit $N_{\max }$ is defined as the number of colony-forming bacterial units (CFU) observed in the vehicle control group in the last $(25 \mathrm{~h})$ readout.

The following first-order growth and second-order kill differential equation model was fit to the data.

$\frac{\mathrm{d} N}{\mathrm{~d} t}=k_{\mathrm{g}} \cdot N \cdot\left(1-\frac{N}{N_{\max }}\right)-k_{\mathrm{k}} \cdot C(t) \cdot N$

$N$ is the number of bacterial counts, $C(t)$ the biophase function, $k_{\mathrm{g}}$ the first-order growth rate constant, and $k_{\mathrm{k}}$ the second-order bacterial kill rate constant. Dose $\mathrm{i}_{\mathrm{i}}$ and $K$ are the drug dose and elimination rate constant, respectively. The (1 $\left.-N / N_{\max }\right)$ term captures the linear reduction in bacterial growth toward the upper limit $N_{\text {max }}$.

The key patterns of this dataset are

- A set predose baseline value and a maximum bacterial growth level

- A first-order growth pattern

- A dose-dependent increase in bacterial kill response followed by regrowth

The key patterns in this case study are observed against a defined vehicle/control response. The onset of drug action becomes shorter and the maximum effect more pronounced with increased dose, suggestive of a main stimulating effect on the loss (= "kill") mechanism. Bacterial regrowth appears as a first-order process $\left(k_{g}\right)$ and occurs in parallel at all dose levels.

\section{Case Study 11}

A new class of agents was developed to irreversibly inhibit an enzyme believed to play a role in many inflammatory disease conditions. High-resolution enzyme activity data were obtained at two dose levels throughout $24 \mathrm{~h}$ (Fig. 9, case study 11 (4)). A faster onset of action to a target response level less than 6 response units was observed with the higher dose. The intensity of response (trough value) was suppressed by $50 \%$ and $90 \%$ after the low and high dose, respectively. The duration of a corresponding clinical response was about 10 and $24 \mathrm{~h}$ in the low- and high-dose groups, respectively. This might suggest that a more than $10-20 \%$ reduction of the enzyme pool (i.e., to $\leq 9-10$ units) is associated with clinical utility.

The buildup of enzyme levels $R$ is produced by a zeroorder turnover rate $k_{\text {in }}$ and eliminated by a first-order process $-k_{\mathrm{out}} \cdot R$, which represents the natural degradation of enzyme. The drug concentration $C$ acts via depletion of $R$, expressed as $-k_{\text {irrev }} \cdot C \cdot R$. The second-order term " $-k_{\text {irrev }} \cdot C \cdot R$ " implies that the total $\mathrm{AUC}_{R}$ will be independent of the shape of the drug concentration-time profile provided the total AUC (drug) stays the same. The turnover of response after an oral dose of drug $\mathrm{X}$ can be written mathematically as 
Case Study 11

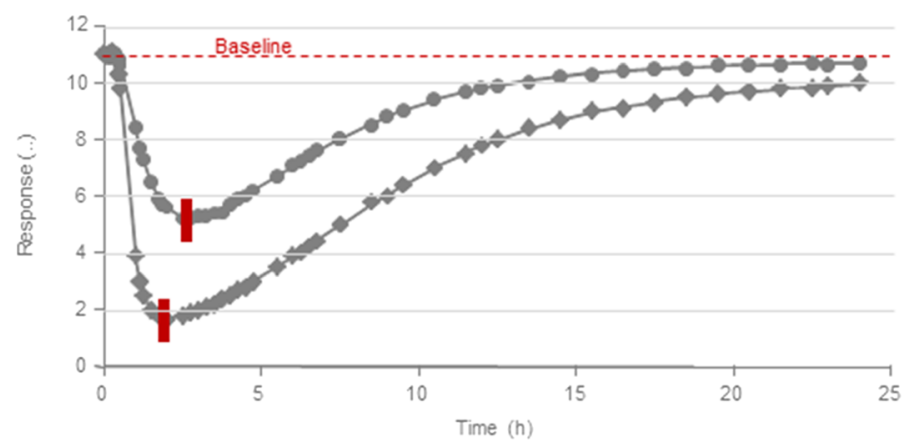

Case Study 13

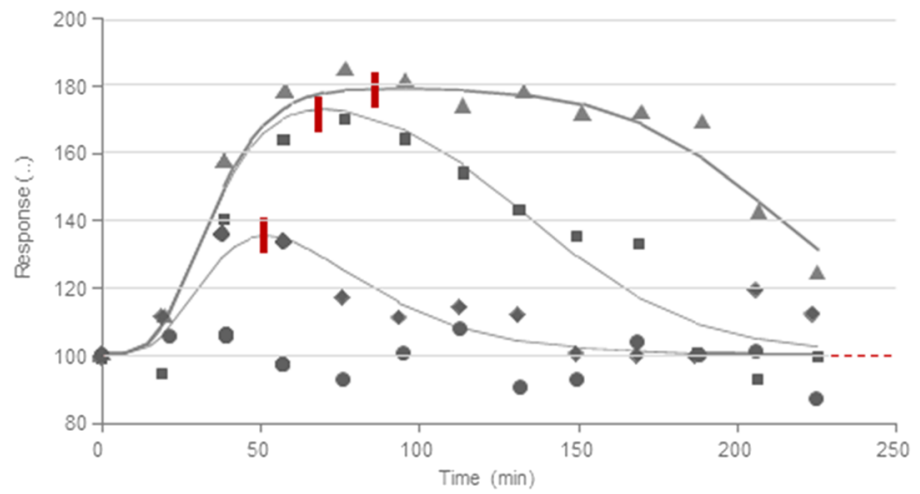

Case Study 12

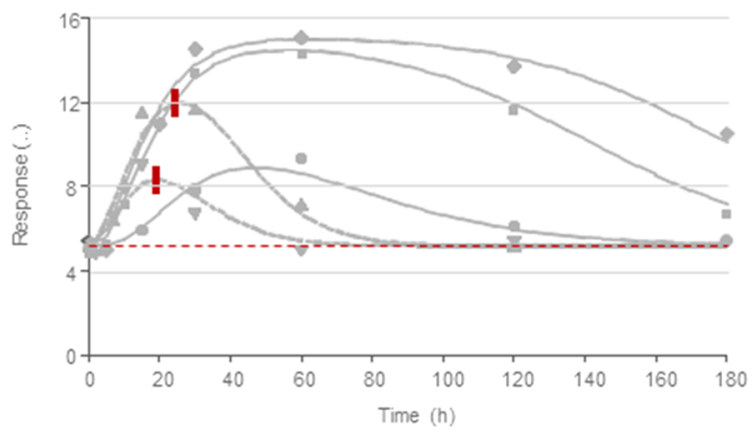

Case Study 14

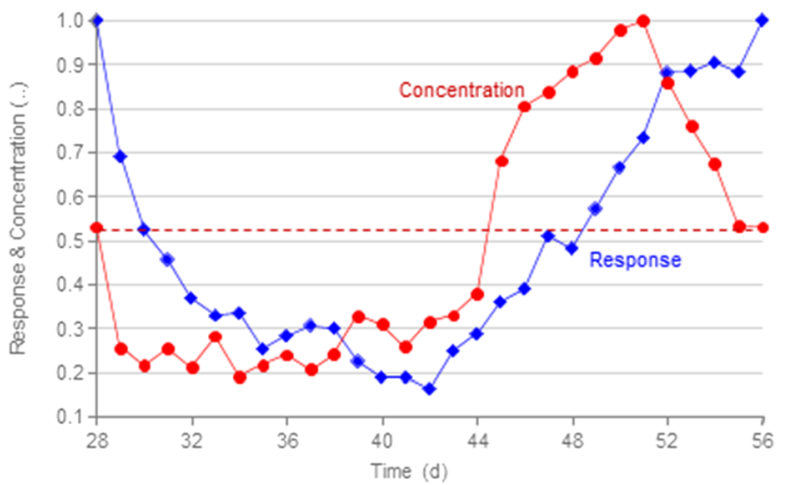

Fig. 9. Case study 11: observed (symbols) and predicted (solid lines) responses from a single-dose study (400 and $1600 \mathrm{mg}$ ). The test compound was given to the same subject at two occasions. Note the shift to the left in the enzyme activity trough value (red bars; decrease in $\left.t_{\max }\right)$ with an increase in dose (4). Case study 12: observed (symbols) and model predicted tail-flick response (lines) vs. time of drug X after different doses given by the intravenous (dashed lines) and subcutaneous (solid lines) routes. The subcutaneous doses are 10,50, and $100 \mu \mathrm{g} \mathrm{kg}{ }^{-1}$ and the intravenous doses are 3 and $10 \mu \mathrm{g} \mathrm{kg}^{-1}$. The red bars show the peak shifts in response with increasing iv doses (4). Case study 13: observed (symbols) and predicted (solid lines) of basal acetylcholine release vs. time after acute dosing at three dose levels (control group, filled triangles; $20 \mu \mathrm{mol} \mathrm{kg}{ }^{-1}$ open circles; $40 \mu \mathrm{mol} \mathrm{kg}{ }^{-1}$ filled circles; $80 \mu \mathrm{mol} \mathrm{kg}{ }^{-1}$ open diamonds) of the nicotinic agonist TC-1734 (21). Red bars denote peak shifts in response with increased doses (4). Case study 14: observed human mediator- (concentration, red) and biomarker- (response, blue) time data. The pattern to observe is that mediator precedes the biomarker response by $4-5$ days. The amplitude in response is about 0.4 units fluctuating from a mean response of about 0.6 units. The response has a cycle period of 28 days

$$
\left\{\begin{array}{l}
\frac{\mathrm{d} R}{\mathrm{~d} t}=k_{\text {in }}-k_{\text {out }} \cdot R-k_{\text {irrev }} \cdot C \cdot R \\
R_{0}=\frac{k_{\text {in }}}{k_{\text {out }}} \\
R_{\mathrm{ss}}=\frac{k_{\text {in }}}{k_{\text {out }}+k_{\text {irrev }} \cdot C_{\mathrm{ss}}}=R_{0} \cdot \frac{1}{1+\frac{k_{\text {irrev }}}{k_{\text {out }}} \cdot C_{\mathrm{ss}}}
\end{array}\right.
$$

where $k_{\text {in }}$ is the turnover rate, $k_{\text {out }}$ the fractional turnover rate, $k_{\text {irrev }}$ the second-order rate constant for the drug response complex $\left(\right.$ time $^{-1} \cdot$ concentration $\left.^{-1}\right)$, and $C$ the concentration of the drug. The equilibrium concentration-response relationship is given by Eq. 28 (bottom line).

The key patterns of this dataset are

- A defined baseline (empirically assumed to be constant)

- A dose-dependent shortening of the onset of action with an increase in dose

- A leftward response trough shift with increasing doses
- A dose-dependent decrease of trough values

- A dose-dependent increase in duration of response

- A parallel return back to baseline in the two responsetime courses

The key patterns in this case study are not observed against a defined vehicle/control response. However, the onset of drug action becomes shorter and the trough response lower with increased dose. The onset of response is rapid and the offset is slow, likely due to slow de novo synthesis of enzyme. Remember that the analysis of response-time data is always improved by concomitant monitoring of the actual exposure profile(s) driving the response.

\section{Case Study 12}

A potential analgesic drug was given at two dose levels ( 1 and $10 \mu \mathrm{g} \mathrm{kg}^{-1}$ ) via the intravenous route and at three dose levels $\left(10,50\right.$, and $\left.100 \mu \mathrm{g} \mathrm{kg}^{-1}\right)$ via the subcutaneous route. The time (in seconds) to respond to a moderate pain stimulus was determined at different times up to $3 \mathrm{~h}$ after dosing. The test model utilizes a reflex response to heat, most commonly induced by focusing a laser beam on the tail of a rodent and 
monitoring the time until the animal withdraws its tail as a measure of pain sensitivity. The tail-flick response-time data were obtained from each dose level after an acute dose (Fig. 9, case study 12 (4)). No plasma exposure data are available and we thus approach the problem by analyzing the available dose-response-time data (22).

A mono-exponential model and a first-order input/ output model are assumed to suitably describe the biophase kinetics after systemic (iv; Eq. 29, upper row) and extravascular (sc; Eq. 29, bottom row) dosing, respectively.

$$
\left\{\begin{array}{l}
A_{\mathrm{b}}=D_{\mathrm{iv}} \cdot\left[e^{-K \cdot t}\right] \\
A_{\mathrm{b}}=\frac{K_{\mathrm{a}} \cdot F^{*} \cdot D_{\mathrm{sc}}}{\left(K_{\mathrm{a}}-K\right)}\left[e^{-K \cdot\left(t-t_{\mathrm{lag}}\right)}-e^{-K_{\mathrm{a}} \cdot\left(t-t_{\mathrm{lag}}\right)}\right]
\end{array}\right.
$$

where $A_{\mathrm{b}}$ denotes the biophase amount, $D_{\text {iv }}$ the intravenous dose, $K$ the elimination rate constant, $K_{\mathrm{a}}$ the absorption rate constant, $F^{*}$ the biophase availability, $D_{\text {sc }}$ the subcutaneous dose, and $t_{\text {lag }}$ the lag time. Note that there are no volume terms in Eq. 29 for the biophase amount expressions. The stimulatory drug "mechanism" function is given by Eq. 30

$S\left(A_{\mathrm{b}}\right)=1+\frac{E_{\mathrm{max}} \cdot A_{\mathrm{b}}^{n}}{\mathrm{ED}_{50}^{n}+A_{\mathrm{b}}^{n}}$

where $E_{\max }, \mathrm{ED}_{50}$, and $n$ are the efficacy, dose at halfmaximal drug-induced response, and the sigmoidicity parameter, respectively.

This nonlinear function causes a peak shift in maximum response with increasing doses and allows the turnover system to capture the time window where saturation occurs in response data. The turnover function of the response where drug acts by stimulating the production of response is

$\left\{\begin{array}{l}\frac{\mathrm{d} R_{1}}{\mathrm{~d} t}=k_{\text {in }} \cdot S\left(A_{\mathrm{b}}\right)-k_{\mathrm{out}} \cdot R_{1} \\ \frac{\mathrm{d} R_{2}}{\mathrm{~d} t}=k_{\text {out }} \cdot R_{1}-k_{\text {out }} \cdot R_{2} \\ \frac{\mathrm{d} R_{\text {obs }}}{\mathrm{d} t}=k_{\text {out }} \cdot R_{2}-k_{\text {out }} \cdot R_{\text {obs }}\end{array}\right.$

The two transit compartments $R_{1}$ and $R_{2}$ are included to capture the slow initial buildup of response $R_{\text {obs }}$. The delay is assumed to primarily reflect the access and buildup of effective analgesic concentrations in the biophase(s) relevant to modulation of the tail-flick response. The predicted response at equilibrium is generated from Eq. 32

$R_{\mathrm{ss}}=\frac{k_{\mathrm{in}}}{k_{\mathrm{out}}} \cdot S\left(A_{\mathrm{b}, \mathrm{ss}}\right)=R_{0} \cdot\left(1+\frac{E_{\mathrm{max}} \cdot A_{\mathrm{b}, \mathrm{ss}}^{n}}{\mathrm{ED}_{50}^{n}+A_{\mathrm{b}, \mathrm{ss}}^{n}}\right)$

$A_{\mathrm{b}, \mathrm{ss}}$ corresponds to the $X$-axis and $R_{\mathrm{ss}}$ to the $Y$-axis.

The key patterns of this dataset are

- A baseline response (defined by the predose pain response threshold in the same animals)

- A slightly delayed onset of action (buildup of response) captured by a series of transit compartments
- A dose-dependent rightward peak shift due to the nonlinear drug "mechanism" function

- Saturation at the highest subcutaneous dose

- Absorption rate-limited drug elimination and thus duration of (subcutaneous) response

- A more rapid decline back to baseline following intravenous compared to subcutaneous dosing

The key patterns in this case study are not observed against a defined separate vehicle/control response, but iv and sc data are compared, and the response-time course is related to the predose pain threshold in the same animals. The onset of drug action exhibits a slight delay followed by a concave buildup. Increasing doses lead to a peak shift in maximum response. Saturation occurs at the highest sc dose and decline of response shows absorption rate-limited elimination.

\section{Case Study 13}

The biomarker data in this case represent acetylcholine (ACh) levels in the cerebral cortex of rats (Fig. 9, case study 13). Single oral administration of a nicotinic agonist test compound elicited a marked increase in the ACh levels in the cerebral cortex, as measured by microdialysis (18). A doseresponse-time effect was observed over the 20 - to $80-\mu \mathrm{mol}-$ $\mathrm{kg}^{-1}$ dose range, with a more sustained duration at higher doses. While tachyphylaxis with ion channel receptors is not uncommon, there was no significant attenuation of the response after 4 days of repeated administration of the test compound, thus fully consistent with the lack of tolerance to the procognitive effects also seen with the drug under these conditions.

Although exposure data from the aforementioned studies are lacking, a model will be proposed that captures the four response-time profiles by means of inclusion of a hypothetical input-output biophase compartment. The assumption is that response-time data contain some kind of "kinetic" information, reflecting the time course of the test compound close to the target in the CNS rather than the plasma kinetics.

We will extend the basic model with a series of transduction steps (Eq. 35). The model contains a first-order input $\left(K_{\mathrm{a}}\right)$-output $(K)$ biophase compartment that captures the turnover of the amount of the test compound. However, as not all parameters in such a biological process chain can be identified and measured, we avoid overparameterization and set $K_{\mathrm{a}}$ equal to $K$, which we then denote $K^{\prime}$ in the model. The latter parameter is part of the regression model parameters. The amount in the biophase $A_{\mathrm{b}}$ is then assumed to drive the receptor pool via a stimulatory function $S\left(A_{\mathrm{b}}\right)$.

The turnover of drug in the biophase was either modeled as a bolus input with first-order loss or as a first-order input/ output model.

$\left\{\begin{array}{l}A_{\mathrm{b}}=\text { Dose } \cdot e^{-K^{\prime} \cdot t} \\ A_{\mathrm{b}}=\frac{\text { Dose } \cdot K_{\mathrm{a}}}{K_{\mathrm{a}}-K} \cdot\left(e^{-K \cdot t}-e^{-K_{\mathrm{a}} \cdot t}\right)\end{array}\right.$

The $K^{\prime}$ parameter is the hypothetical elimination rate constant of the drug from the biophase, and $K_{\mathrm{a}}$ is the first- 
order input rate constant to the biophase compartment. The stimulatory drug "mechanism" function $S\left(A_{\mathrm{b}}\right)$ acting on the receptor pool is given by Eq. 34 as

$S\left(A_{b}\right)=1+\frac{S_{\max } \cdot A_{b}^{n}}{S D_{50}^{n}+A_{b}^{n}}$

$A_{\mathrm{b}}$ is the amount of drug in the biophase, $S_{\max }$ the maximum drug-induced stimulation of the turnover rate of $R_{1}, \mathrm{SD}_{50}$ the amount in biophase giving $50 \%$ of maximum stimulation, and $n$ the dose-response curve sigmoidicity factor. The turnover of action in the various receptor, transducer, and response compartments is mathematically written as

$$
\left\{\begin{array}{l}
\frac{\mathrm{d} R_{1}}{\mathrm{~d} t}=\frac{1}{\tau} \cdot\left(R_{0} \cdot S\left(A_{\mathrm{b}}\right)-R_{1}\right) \\
\frac{\mathrm{d} R_{2}}{\mathrm{~d} t}=\frac{1}{\tau} \cdot\left(R_{1}-R_{2}\right) \\
\frac{\mathrm{d} \dot{R}_{i-1}}{d t}=\frac{1}{\tau} \cdot\left(R_{i-2}-R_{i-1}\right) \\
\frac{\mathrm{d} R_{i}}{\mathrm{~d} t}=\frac{1}{\tau} \cdot\left(R_{i-1}-R_{i}\right)
\end{array}\right.
$$

where $\tau$ is the transit time, $R_{0}$ the baseline response level, and $R_{1}$ the receptor variable. Our model includes a receptor compartment where the stimulation of turnover rate occurs, five transducer compartments mimicking the extended delay of response, and a terminal response compartment $R_{i}$. Since we have a dataset covering the baseline behavior during $225 \mathrm{~min}$, we will also use those data in the model. The equilibrium biophase amount-response relationship is given by Eq. 36.

$R_{\mathrm{ss}}=R_{0} \cdot S\left(A_{\mathrm{b}}\right)=R_{0} \cdot\left(1+\frac{S_{\max } \cdot A_{\mathrm{b}}^{n}}{\mathrm{SD}_{50}^{n} \cdot A_{\mathrm{b}}^{n}}\right)$

The key patterns of this dataset are

- A stable baseline (obtained from experimental data in the same study)

- A small initial delay (15 min), possibly due to distributional factors

- A concave time course of onset of action

- A dose-dependent peak shift and duration of response

- A flat portion at the highest dose

The concave onset may imply some kind of transduction process (the buildup of response increases exponentially after a brief lag time). The peak shifts and saturable top dose-response are strong indicators of nonlinear stimulation. The reasoning and modeling of this example would appear to be a very plausible approach, accounting for the shape of the kineticl dynamic response relation by the introduction of a series of compartmental transduction delays. Hence, the outcome would be explainable in credible physiological/biological terms. However, before embarking on an elaborate modeling effort, it is advisable always to make a "reality" check! Thus, in this particular instance, there is substantial reason to believe that a major part of the explanation rather results from methodological factors. Thus, the microdialysis membrane used in the study contains permanently negatively charged groups. This property will strongly affect the extraction dynamics of compounds that are positively charged (at physiological $\mathrm{pH}$, or otherwise) - in essence because the membrane acts as an ion-exchange column under these conditions (23). This, in turn may well explain the initial "concave" portion as well as the protracted washout part of the ACh response curve following drug administration. Therefore, even if the biological interpretation underlying the model in this very case may not accurately reflect the pharmacodynamic outcome, there may well be other situations in which the initially referred "transduction process" reasoning is much closer to the causal factors.

\section{Case Study 14}

This example contains information from an anonymized set of data obtained in studies of the infradian relation between a hormone and a downstream biomarker in man. The oscillatory pattern in the biomarker response was mirrored by variations in an endogenous mediator hormone that also displayed a 28-day cycle between peak values. The amplitude of the biomarker response was about 0.4 units with a peak at 1 (unity) and a trough of 0.2 . The role of the mediator hormone is considered to act as a stimulator of production of the biomarker response, which appears to be delayed by approximately $4-5$ days relative to the mediator concentration (Fig. 9, case study 14 (4)).

The plasma concentration-time course of mediator is given as a table function, which means that a table of the actual plasma concentrations is input into the drug "mechanism" function. Concentration-time values between the actual observations are linearly interpolated.

$C_{i}=f($ table $)$

The nonlinear stimulatory mediator "mechanism" function $S(C)$ is defined as

$S(C)=1+\frac{S_{\max } \cdot C^{n}}{E C_{50}^{n} \cdot C^{n}}$

The mediator is assumed to directly "drive" or stimulate factors responsible for production of the biomarker response via a nonlinear function (Eq. 39, third row).

$$
\left\{\begin{array}{l}
\frac{\mathrm{d} R_{1}}{\mathrm{~d} t}=k_{\text {in }} \cdot S(C)-k_{\text {out }} \cdot R_{1} \\
\frac{\mathrm{d} R_{2}}{\mathrm{~d} t}=k_{\text {out }} \cdot R_{1}-k_{\text {out }} \cdot R_{2} \\
S(C)=1+\frac{S_{\max } \cdot C^{n}}{\mathrm{SC}_{50}^{n}+C^{n}} \\
R_{\mathrm{ss}}=R_{02} \cdot S(C)=R_{02} \cdot\left(1+\frac{S_{\max } \cdot C^{n}}{\mathrm{SC}_{50}^{n}+C^{n}}\right)
\end{array}\right.
$$

$R_{2}$ in Eq. 39 (second row) is the observed biomarker response and $R_{02}$ the baseline value. A single turnover compartment did not adequately account for the 4-5-day time delay between plasma concentrations of the hormonal mediator $C$ and the biomarker response. Since the 
endogenous levels of the mediator $C$ are oscillating over a 28day cycle, $S(C)$ and the biomarker readout $R$ will also oscillate. The equilibrium hormone concentration-biomarker response relationship is shown in Eq. 39 (bottom row) where $R_{0}$ stands for the averaged baseline readout across the full 28day cycle, $S_{\max }$ the efficiency parameter, $\mathrm{SC}_{50}$ the potency, $n$ the response curve sigmoidicity parameter, and $C$ the concentration of the hormonal mediator in plasma.

The key patterns of this dataset are

- An oscillating baseline response driven by an oscillating hormone concentration across a 28-day infradian cycle

- A 4-5-day shift between hormone concentration and biomarker response

- An up- and downward response variation amplitude of 0.6 units across the 28-day cycle

The presented pattern is thus an example of a response which oscillates across a set cycle time, with a clearly observable delay between the mediator concentration and biomarker response readout. Despite a seemingly protracted trough in the mediator levels in this example, most of the biomarker response curve remains separated from its nadir response (0.2). This suggests that the correlation between the two variables may be best captured by a nonlinear saturable drug "mechanism" function.

\section{Case Study 15}

Quantitative pharmacodynamic information on the cortisol response was derived from a study using a constant rate infusion regimen of dexamethasone $(0.17,1.7$, and $17 \mu \mathrm{g} \mathrm{kg}^{-1}$ ) to six standardbred horses. An oscillatory turnover rate based on a cosine function (amplitude and cycle) captured the oscillatory behavior in data well when simultaneously fitted to all dose groups. Turnover rate and fractional turnover rate and the drug (potency, efficacy, and sigmoidicity parameters) properties were quantified for all horses. Representative data for one horse are shown in Fig. 10 (case study 15 (24)).

The synthetic glucocorticoid dexamethasone is assumed to directly inhibit the endogenous production of cortisol. A turnover rate equation may therefore be formulated where the drug "mechanism" function is described as

$I(C)=1-\frac{I_{\max } \cdot C^{n}}{\mathrm{IC}_{50}^{n}+C^{n}}$

where $I(C)$ is the inhibitory drug "mechanism" function, $I_{\max }$ the maximum drug-induced inhibition of cortisol, $\mathrm{IC}_{50}$ the dexamethasone plasma concentration at $50 \%$ reduction of the cortisol turnover rate, and $n$ the exposure-response curve sigmoidicity factor. The turnover of hydrocortisone with the inhibitory drug "mechanism" incorporated (Fig. 10, case study 15) is thus described by

$$
\left\{\begin{array}{l}
\frac{\mathrm{d} R_{1}}{\mathrm{~d} t}=k_{\text {in }}(t) \cdot I(C)-k_{\text {out }} \cdot R_{1} \\
\frac{\mathrm{d} R_{2}}{\mathrm{~d} t}=k_{\text {out }} \cdot R_{1}-k_{\text {out }} \cdot R_{2} \\
R_{0}=\frac{k_{\text {in }}}{k_{\text {out }}}
\end{array}\right.
$$

where $\mathrm{d} R_{1} / \mathrm{d} t$ and $\mathrm{d} R_{2} / \mathrm{d} t$ are the rate of change of response in $R_{1}$ and $R_{2}$, respectively. $R_{1}$ is associated with the drug action in the biophase and $R_{2}$ the downstream response, i.e., cortisol in plasma. The terms $k_{\text {in }}(t)$ and $k_{\text {out }}$ denote the time-dependent turnover rate and the first-order fractional turnover rate, respectively. Cortisol is released into plasma in response to a circadian input process, and the (baseline) circadian turnover rate $k_{\text {in }}(t)$ thus approximated by a cosine function described as

$k_{\text {in }}(t)=k_{\text {in,mean }}+\alpha \cdot \cos \left(\frac{2 \pi}{24} \cdot\left(t=t_{0}\right)\right)$

where $k_{\text {mean }}$ is the mean input rate, response amplitude, and $t$ and $t_{0}$ the clock time within the 24 -h cycle and peak time, respectively. The ratio term $2 \cdot \pi / 24$ converts the 24 -h period into radians. An absolute response amplitude of about 20 units is identified from baseline data which has a mean of about 60 and oscillates between 40 and 80 response units over a 24--cycle. The time-dependent response baseline is given as

$R_{0}(t)=\frac{k_{\text {in }}(t)}{k_{\text {out }}}$

\section{The key patterns of this dataset consist of}

- A natural oscillating baseline which defines the response amplitude and shape

- A clear-cut dose separation in the response-time courses with complete response suppression approaching zero at the highest dose

- A dose-dependent separation in the duration of suppressed response, followed by recovery to the natural baseline oscillations

Since the background natural oscillating baseline differs from zero, the response can be parameterized including both the turnover (= production) rate (in this case a function) and fractional turnover (=loss) rate. A nonlinear inhibitory drug mechanism function captured the 100-fold dose range better than a linear function since the biomarker responses were simultaneously fit for all dose levels.

\section{Case Study 16}

The antilipolytic response in a group of healthy volunteers was followed in plasma after a constant rate intravenous infusion over $6 \mathrm{~h}$ of the adenosine A1 receptor agonist ARA (Fig. 10, case study 16). The response (levels of free fatty acids, FFA) started at a baseline and declined to a trough value at about $2 \mathrm{~h}$ after the start of the constant rate infusion. The response then gradually returned back toward the baseline in spite of a stable (or even slightly mounting) drug exposure, interpreted to reflect functional adaptation (tolerance). Upon cessation of the constant rate infusion at $6 \mathrm{~h}$, the response also displayed a rebound (increase above the baseline). A pool/ precursor model was suggested by the authors to account for the overall shape of the response (25). This type of model aims to describe drug action on the linkage between two serially coupled production/loss "tanks" (cf. Fig. 2), rather than an effect on turnover/fractional turnover in either of the two. 
Case Study 15

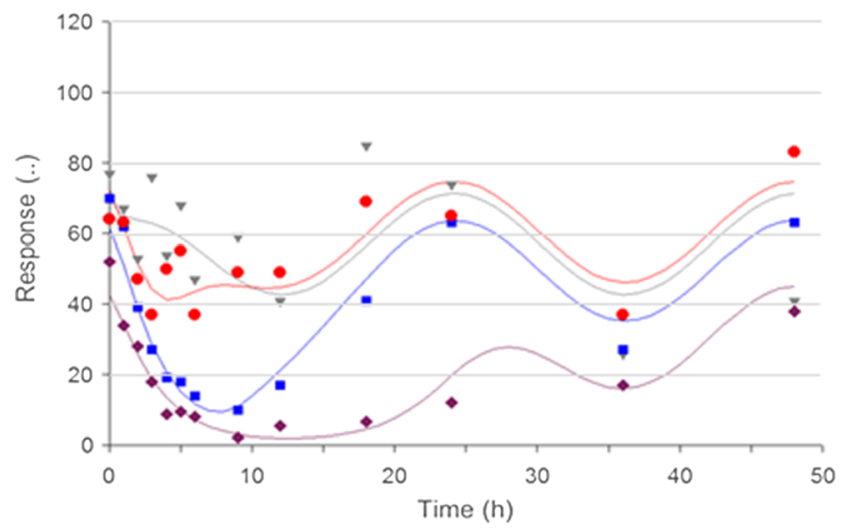

Case Study 17

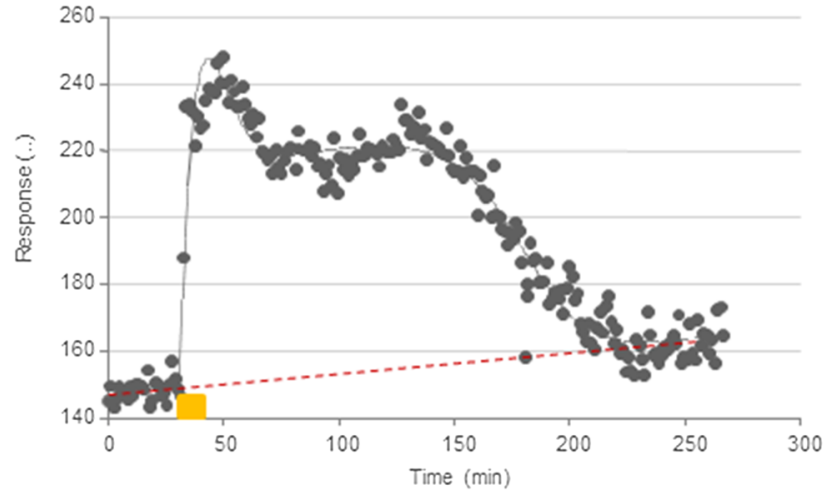

Case Study 16

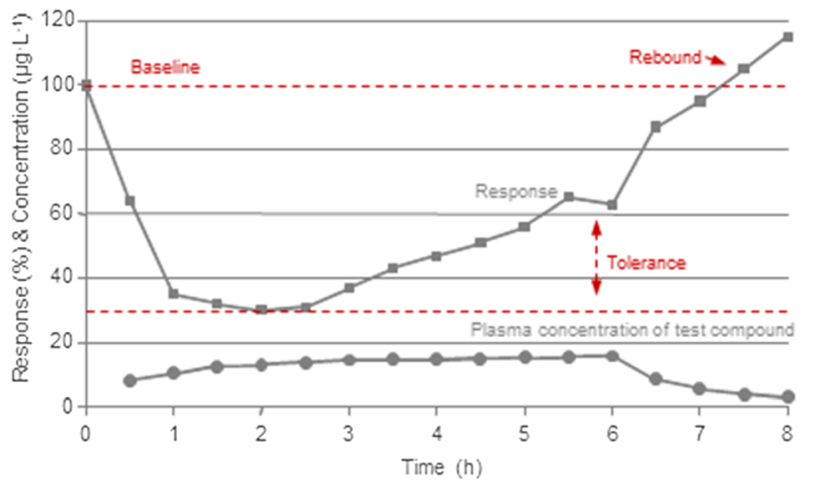

Case Study 18

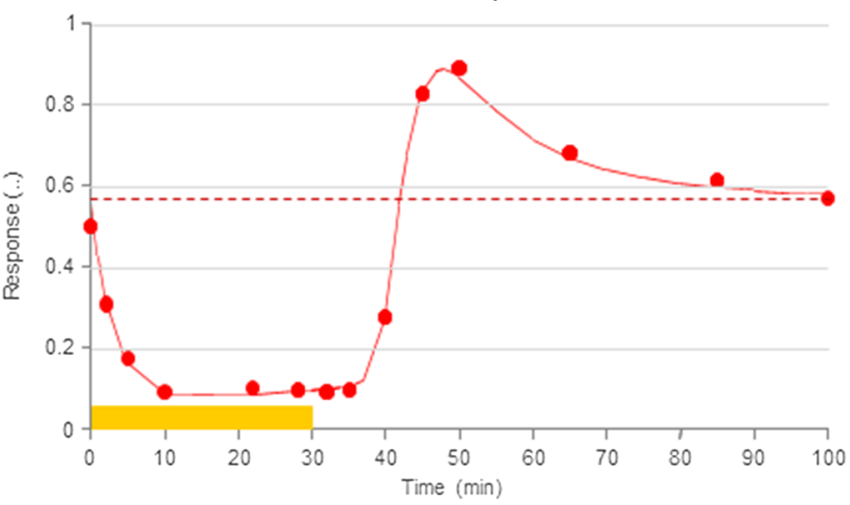

Fig. 10. Case study 15: observed (filled symbols) and model predicted (lines) response-time data following a bolus + infusion regimen of dexamethasone to a horse. Shown are baseline data obtained from vehicle dosing $(0 \mathrm{mg}$ solid down triangles and gray line), low dose $\left(0.17 \mu \mathrm{g} \mathrm{kg}^{-1}\right.$, red dots and line $)$, intermediate dose $\left(1.7 \mu \mathrm{g} \mathrm{kg}^{-1}\right.$, blue squares and line $)$, and high dose $\left(17 \mu \mathrm{g} \mathrm{kg}^{-1}\right.$, purple diamonds and line). Data are obtained from Ekstrand et al. (24). Case study 16: observed antilipolytic response (filled squares) and plasma concentration (filled circles) data of Zannikos et al. (25) during and after a constant rate intravenous infusion of the test compound (13). Case study 17: observed (filled symbols) and model predicted (gray line) EEG effect-time data following an intravenous infusion of compound X. The drug infusion starts at $30 \mathrm{~min}$ and stops at $40 \mathrm{~min}$. The dashed red line shows the baseline drift. The yellow bar shows the length of infusion. Case study 18: free fatty acid FFA response $v s$. time. Observed (symbols) and model predicted (line) response-time data in one rat after constant rate infusion of NiAc for $30 \mathrm{~min}$ indicated by the yellow horizontal bar (Isaksson et al. (26))

This profile then served as input to the nonlinear inhibitory "mechanism" function, lowering the FFA concentrations

$I(C)=1-\frac{I_{\max } \cdot C^{n}}{\mathrm{IC}_{50}^{n}+C^{n}}$

where $\mathrm{IC}_{50}$ and $n$ denote the potency and the sigmoidicity factor of the drug, respectively. This inhibitory drug "mechanism" function links the kinetics to the dynamics. Hence, the turnover of the pool/precursor $P$ compartment becomes

$$
\left\{\begin{array}{l}
\frac{\mathrm{d} P}{\mathrm{~d} t}=k_{\text {in }}-k_{\mathrm{out}} \cdot P \cdot I(C) \\
P_{0}=\frac{k_{\text {in }}}{k_{\mathrm{out}}} \\
I(C)=1-\frac{I_{\text {max }} \cdot C^{n}}{\mathrm{IC}_{50}^{n}+C^{n}} \\
P_{\mathrm{ss}}=\frac{k_{\text {in }}}{k_{\mathrm{out}} \cdot I(C)}
\end{array}\right.
$$

where $k_{\text {in }}$ and $k_{\text {out }}$ are the turnover rate and fractional turnover rate of the response, respectively. The turnover of the response compartment, baseline response, and steadystate response becomes

$$
\left\{\begin{aligned}
\frac{\mathrm{d} R}{\mathrm{~d} t} & =k_{\text {out }} \cdot P \cdot I(C)-k_{\text {out }} \cdot R \\
R_{0} & =\frac{k_{\text {in }}}{k_{\text {out }}} \\
R_{\text {ss }} & =\frac{k_{\text {in }}}{k_{\text {out }}}
\end{aligned}\right.
$$

Equation 46 (bottom row) shows that the response is independent of drug exposure at steady state and returns to the baseline value $k_{\text {in }} / k_{\text {out }}$. This is a landmark when using the pool/precursor turnover model for modeling acute dosing data. Note also that we have used the same rate constant $k_{\text {out }}$ from the pool and response compartments due to parameter redundancy. 
The key features of this pattern analysis are

- A predose baseline

- Pool/precursor-driven adaptation at constant or rising plasma exposure

- A post-infusion rebound effect

The key patterns in this case study are not observed against a defined vehicle/control response. The response displays a clear adaptation in spite of constant or increasing plasma concentrations of the test compound. A standard pool/ precursor model is applied in the original publication. A slight tendency of rebound is observed for the terminal observation(s), but as the time course is truncated, the exact extent of rebound cannot be determined from the data.

\section{Case Study 17}

This case study aims at showing how to model a pharmacological response biomarker ( $E E G$ signal) measured in rats during and after a 10-min constant intravenous infusion of a new experimental agonist $A$ (Fig. 10, case study 17). The biomarker displays an intriguingly complex behavior with a rapid rise, an overshoot outlasting the infusion period, return toward an apparent pharmacodynamic steady state, then followed by a slower decline back to the baseline. The baseline exhibits a slight drift over time, which will be tackled by means of a time-dependent turnover rate $k_{\text {in }}(t)$ in the analysis.

To account for the observed pattern, we assume a negative feedback system such as the one schematically illustrated in Fig. 3 (case study 17 (4)). Whatever happens in or with the response $R$ compartment will eventually also indirectly affect a moderator $M$ compartment. The moderator compartment in turn has its own rate constant $k_{\text {tol }}$ and kinetics of turnover that control the rate of "tolerance" development. ${ }^{3}$ The change in the tolerance moderator level $M$ will eventually also affect either the buildup of $R$ via $k_{\text {in }}$ or the loss of $R$ via $k_{\text {out }}$. In the present case, we assume that the moderator regulates $R$ via an inhibitory effect of $k_{\text {in }}$, i.e., negative feedback.

Directly after the start of infusion ( $\sim 30 \mathrm{~min})$ of the test compound, the response increased rapidly from about 150 units up to approximately 230 units at the end of infusion ( $\sim 40 \mathrm{~min}$ ). Observe the rapid upswing, the overshoot despite stop of drug infusion, the peak response lasting for $\sim 30 \mathrm{~min}$ post-infusion, the prolonged relatively stable response ("roof of the cathedral") for yet another $\sim 80 \mathrm{~min}$, and thereafter a slow downswing to baseline (at $\sim 210 \mathrm{~min}$ ) without observable rebound. This unusual pattern may be conceptualized in biological terms by assuming that the drug upon reaching a certain threshold level will also trigger a negative feedback modulator/process that takes the edge off the response for a period of time (50-150 min), until the drug levels again are below concentrations required to activate this dampening factor or process. The EEG effects of compound $\mathrm{X}$ in the rats are modeled by means of a system of differential Eq. 47,

${ }^{3}$ It is beyond the scope of the present framework to discuss the concept of tolerance broadly. However, suffice it to say that the expression in this context is used to cover various modes of counterregulatory and adaptive processes that serve to diminish the response to a drug, be it upon acute or repeated administration. where $I(C)$ is the inhibitory drug "mechanism" function-that is, the feedback modulator/process.

$\left\{\begin{array}{l}\frac{\mathrm{d} R}{\mathrm{~d} t}=\frac{k_{\mathrm{in}}(t)}{M}-k_{\mathrm{out}} \cdot R \cdot I(C) \\ I(C)=1-\frac{I_{\max } \cdot C^{n}}{\mathrm{IC}_{50}^{n}+C^{n}} \\ \frac{\mathrm{d} M}{\mathrm{~d} t}=k_{\mathrm{tol}} \cdot R-k_{\mathrm{tol}} \cdot M\end{array}\right.$

One may also want to apply a model with stimulation $S(C)$ of the turnover rate $k_{\mathrm{in}}$.

$\left\{\begin{array}{l}\frac{\mathrm{d} R}{\mathrm{~d} t}=\frac{k_{\mathrm{in}}(t)}{M} \cdot S(C)-k_{\mathrm{out}} \cdot R \\ S(C)=1+\frac{S_{\max } \cdot C^{n}}{\mathrm{IC}_{50}^{n}+C^{n}} \\ \frac{\mathrm{d} M}{\mathrm{~d} t}=k_{\mathrm{tol}} \cdot R-k_{\mathrm{tol}} \cdot M\end{array}\right.$

The concentration-time data $C$ are modeled by means of a two-compartment linear model. At pharmacodynamic steady state and with inhibition of $k_{\text {out }}$ or stimulation of $k_{i n}$, the response becomes

$\left\{\begin{array}{l}R_{\mathrm{ss}}=\sqrt{\frac{k_{\mathrm{in}}}{k_{\mathrm{out}}}} \cdot \sqrt{\frac{1}{I(C)}}=R_{0} \cdot \sqrt{\frac{1}{I(C)}} \\ R_{\mathrm{ss}}=\sqrt{\frac{k_{\mathrm{in}}}{k_{\mathrm{out}}}} \cdot \sqrt{S(C)}=R_{0} \cdot \sqrt{S(C)}\end{array}\right.$

The time course of drift in baseline was modeled by means of a time-dependent change in the turnover rate $k_{\text {in }}(t)$ in Eqs. 47 and 48 (top lines) where $k_{\text {in }}(t)$ is modeled according to

$k_{\text {in }}(t)=k_{\text {in }}(0)+\alpha \cdot t$

\section{The key patterns of this dataset are}

- A time-dependent drift in baseline (approximated by visually comparing flat portions of the time-response curves before drug vs. end of experiment; the study lacks a separate baseline experiment)

- A rapid onset of action (upon the 10-min intravenous infusion) reaching a peak of response followed by

- An early response overshoot despite cessation of the infusion

- A rapid decline down to and apparently constant response for about $80 \mathrm{~min}$

- No observable rebound

At about $150 \mathrm{~min}$, the response-time trajectory assumes a steady rate of decline toward the baseline without displaying any rebound effects. Also note the substantial drift in the baseline with time. This was tackled by a time-dependent turnover rate which increases linearly with time. The unusual response pattern captured by the modeling approach described also helped in creating testable hypotheses on the role and 
impact of the underlying modulator/process involved in the EEG response observed and what is achievable in response during extended exposure.

\section{Case Study 18}

This case study shows data obtained from an acute constant rate infusion experiment of the antiatherogenic, lipid-lowering GPR109A (HM74A) agonist nicotinic acid (NiAc) in rats and simultaneously monitoring the time course of plasma nonesterified FFA (pharmacodynamic response biomarker) levels (Fig. 10, case study 18 (26). Activation of GPR109A in adipose tissue leads to reduced lipolysis and in turn to decreased release of FFA into plasma. The response remains suppressed for a little less than $10 \mathrm{~min}$ after the stop of infusion. This observation is interpreted to indicate that the NiAc exposure remains at a level still sufficient to achieve significant GPR109A-induced restraint of adipose lipolysis, as reflected in low plasma levels of FFA. The subsequent return of FFA toward the baseline $(\sim 10 \mathrm{~min})$ following cessation of NiAc infusion is rapid and followed by a substantial rebound "overshoot" effect (area of rebound is approximately $40 \%$ of the area of FFA suppression), that returns to baseline within the subsequent $60 \mathrm{~min}$.

Figure 11 is a plot of the exposure relative to the FFA response during the experiment, showing a clockwise hysteresis during the constant rate $\mathrm{NiAc}$ infusion. Between 5 and $28 \mathrm{~min}$, there is very little change in the plasma concentration, but the FFA response is nonetheless somewhat further suppressed. The NiAc infusion is stopped at $30 \mathrm{~min}$ and a rapid drop in exposure to test compound ensues. The rebound response occurs when test compound concentration has fallen below its concentration range for significant pharmacodynamic effect, some time between 40 and $45 \mathrm{~min}$. The clockwise hysteresis observed during high drug exposure changes direction to an anticlockwise hysteresis upon drug washout concomitant with the display of rebound.

A one-compartment model with an endogenous turnover rate (endogenous production of $\mathrm{NiAc}$ ) was fitted to $\mathrm{NiAc}$ plasma concentration-time data. Clearance $\mathrm{Cl}$ and volume of distribution $V$ were estimated to $0.099 \mathrm{~L} \mathrm{~min}^{-1} \mathrm{~kg}^{-1}$ and

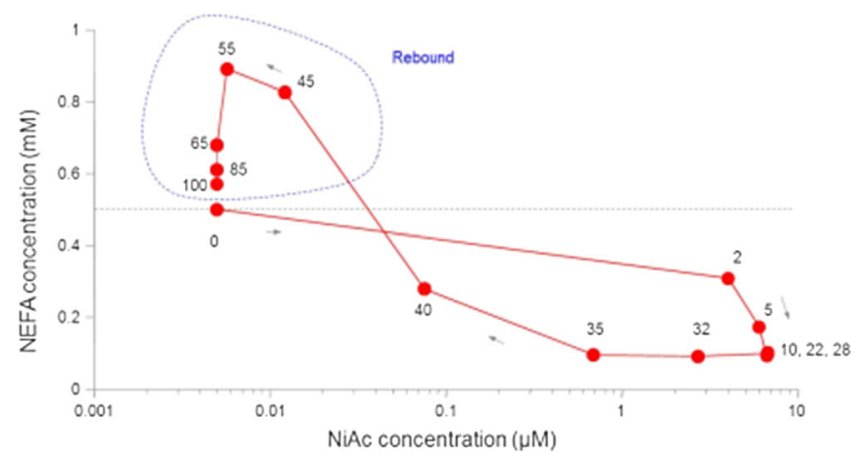

Fig. 11. NiAc concentration- fatty acid (FFA) response plot of data from case study 18 in time order during and after a 30-min constant rate intravenous infusion to rats. The numbers indicate time points and arrows show the time order of events
$0.23 \mathrm{~L} \mathrm{~kg}^{-1}$, respectively. $I(C)$ represents the drug "mechanism" function of NiAc that is inhibitory upon the production of FFA (response $R$ )

$I(C)=1-\frac{I_{\max } \cdot C^{n}}{\mathrm{IC}_{50}^{n}+C^{n}}$

where $C$ is the plasma concentration of NiAc, $\mathrm{IC}_{50}$ the plasma concentration of NiAc that inhibits the production of FFA by $50 \%$ (potency), $I_{\max }$ the maximum FFA-inhibitory capacity, and $n$ the exposure-response sigmoidicity parameter. It is assumed that the activation and loss of FFA response $R$ can be modeled by the following relationship

$$
\left\{\begin{array}{l}
\frac{\mathrm{d} R}{\mathrm{~d} t}=k_{\mathrm{in}} \cdot I(C) \cdot \frac{R_{0}}{M}-k_{\mathrm{out}} \cdot R \\
\frac{\mathrm{d} M}{\mathrm{~d} t}=k_{\mathrm{tol}} \cdot R-k_{\mathrm{tol}} \cdot M \\
R_{0}=\frac{k_{\mathrm{in}}}{k_{\mathrm{out}}}
\end{array}\right.
$$

NiAc inhibits the turnover rate $k_{\text {in }}$ of FFA via the drug "mechanism" function $I(C)$. That is to say that the net downstream result of NiAc agonistic action on GPR109A (HM74) receptors is a reduction of the conversion of adipose triglycerides into FFA (concomitantly released into plasma, the matrix used to monitor the response). Changes in FFA are also counterbalanced by an endogenous modulator, denoted $M$. Note that the loss of $R$ is indirectly governed by means of $M$. The production of $M$ is hence controlled by $R$ and the rate constant for turnover of $M$. The $k_{\text {tol }}$ parameter corresponds to development of tolerance to the drug effect. The $k_{\text {tol }}$ parameter is assumed to govern both production and elimination of $M$ in this particular example because $M$ is not measured per se.

The key patterns of this dataset are

- A predose baseline

- A rapid initial drop in response (due to short half-life of FFA: (26))

- A suppression of response throughout the duration of the 30-min infusion

- A sustained suppression for almost 10 min after stop of infusion

- A rapid recovery of response thereafter

- A substantial rebound "overshoot" which then slowly returns back to baseline within an hour from the peak rebound

Analysis of the exposure-response curves shows a time delay between concentration and response-time courses and a saturation of response at about $1 \mu M$ exposure to drug. Only one infusion regimen is applied so it is not possible to judge about peak shifts at lower or higher infusion rates. Interestingly, the return from overshoot is slower than the initial drug-induced drop in levels. It appears unlikely that the plasma half-life of the FFA would have changed during the experiment. The observed difference in slopes therefore suggests that it is the generation and/or release of the FFA $k_{\text {in }}$ that 
has become transiently upregulated by the initial NiAc-induced inhibition of its formation.

\section{Case Study 19}

The data for this example is taken from an experiment whereby the effect of an anonymized test compound upon the gene expression for a specific target was studied. The pharmacodynamic target data (fold induction of messenger RNA (mRNA)) are shown in Fig. 11 (case study 19) after two single oral doses of test compound $\mathrm{X}$. There is an initial peak at 2-3 $\mathrm{h}$ followed by a downswing that passes the baseline at 6-9 $\mathrm{h}$, then reaching the maximum rebound at $11-14 \mathrm{~h}$ before gradually again returning to the baseline at 30-36 h. The duration of the observed response clearly outlasts the plasma exposure of the (relatively short half-life) compound. A reasonable assumption is that this reflects an action of the drug which serves to trigger a series of processes that due to underlying physiology displays more protracted buildup and waning phases. The higher dose of the drug reaches maximum response later and also rebounds later and deeper. Note the semilogarithmic scale of mRNA induction which more clearly displays the rebound of mRNA data starting at 7 and $10 \mathrm{~h}$ after the low and high dose, respectively.

The exposure profiles of the test compound served as a "driver" of the stimulatory effect $S(C)$ on the turnover rate of mRNA. The turnover equations for $R$ (fold mRNA induction) and $M$ (moderator) are given by the system of differential equations (Eq. 53),

$$
\left\{\begin{array}{l}
\frac{\mathrm{d} R}{\mathrm{~d} t}=k_{\mathrm{in}} \cdot S(C) \cdot\left(\frac{R_{0}}{M_{2}}\right)-k_{\mathrm{out}} \cdot R \\
\frac{\mathrm{d} M_{1}}{\mathrm{~d} t}=k_{\mathrm{tol}} \cdot R_{1}-k_{\mathrm{tol}} \cdot M_{1} \\
\frac{\mathrm{d} M_{2}}{\mathrm{~d} t}=k_{\mathrm{tol}} \cdot M_{1}-k_{\mathrm{tol}} \cdot M_{2}
\end{array}\right.
$$

where $k_{\text {in }}$ is the turnover rate and $k_{\text {out }}$ the fractional turnover rate of $R$ and $k_{\text {tol }}$ the fractional turnover rate of $M$. Note that a sequence of transit compartments of $R$ and $M$ was selected in order to capture the initial concave rise over time of fold mRNA data. The initial conditions of Eq. 53, $R_{0}, M_{01}$, and $M_{02}$, of $R$ and $M$ have the same value (1, unity). The relation between $k_{\text {in }}$ and $k_{\text {out }}$ when no drug induced stimulation occurs is defined by Eq. 54 .

$k_{\text {in }}=k_{\text {out }} \cdot R_{0}$

Each parameter in the model is estimated graphically from experimental data. An approximate initial estimate of $k_{\text {out }}$ is obtained from the assumption that two transit compartments are required to capture the initial time delay in the mRNA response.

The key patterns of this dataset are

- A defined predose baseline (obtained in a separate study)

- A rapid rise in the onset of action

- A dose-dependent peak shift followed by a rapid decline and a rebound at both dose levels that is deeper and shifted toward later time points with the higher dose
The peak shift indicates nonlinear stimulation of the gene transcription $(m R N A)$ response. The overall curve shapes, including post-peak rebound, suggest the presence of a negative modulator mechanism/process-e.g., endproduct inhibition-that serves to keep the net gene product output within a preset physiological frame relative to demand over time.

\section{Case Study 20}

This set of data deals with the acute effect of an antidepressant agent on the extracellular levels of the neurotransmitter serotonin. The original data are obtained from Bundgaard et al. (27). The model was extended to incorporate a two-step moderator transduction model (28). Response-time data are shown in Fig. 12 (case study 20) after three 60-min constant rate intravenous infusions (solid gray bar) and washout.

Escitalopram is a selective serotonin (5-hydroxytryptamine, 5-HT) reuptake inhibitor (SSRI) exhibiting antidepressant and anxiolytic activities in preclinical and clinical studies. Acutely, SSRIs selectively block neuronal 5-HT reuptake, in turn resulting in increased concentrations of the neurotransmitter at the serotonergic cell body and nerve terminal level. This SSRI-induced increase will enhance the serotoninergic transmission. However, it is counteracted initially by the accompanying 5-HT-elicited activation of neuronal negative feedback processes that reduce neuronal firing, synthesis, and release of new 5-HT into the synapse. This limits the acute action of SSRIs on 5-HT neurotransmission and has been hypothesized to contribute to the delayed clinical efficacy of such agents (cf. 29-31). Upon repeated SSRI treatment, the negative feedback is then attenuated and the serotonergic firing rate and 5-HT release is restored. In turn, this now "unlocks" the effect of an SSRI on overall serotonergic neurotransmission. The aforementioned negative feedback derives from several different receptor populations and may therefore for modeling purposes be amalgamated into a single moderator compartment (27). However, empirical studies later found that the suboptimal precision in potency derived from such an approach could be greatly increased by introducing a second moderator (28). The data are thus modeled by applying a two-step series of moderator compartments $M_{1}$ and $M_{2}$.

The regression of response-time data was done using the following turnover equation:

$\left\{\begin{array}{l}\frac{\mathrm{d} R}{\mathrm{~d} t}=\frac{k_{\mathrm{in}}}{M_{2}}-k_{\mathrm{out}} \cdot R \cdot I(C) \\ \frac{\mathrm{d} M_{1}}{\mathrm{~d} t}=k_{\mathrm{out}} \cdot I(C) \cdot R-k_{\mathrm{tol}} \cdot M_{1} \\ \frac{\mathrm{d} M_{2}}{\mathrm{~d} t}=k_{\mathrm{tol}} \cdot M_{1}-k_{\mathrm{tol}} \cdot M_{2}\end{array}\right.$

Here, $R$ is the pharmacodynamic response, $k_{\text {in }}$ the turnover rate (basal level of serotonin), $M_{1}$ and $M_{2}$ the first and second moderator compartments, $k_{\text {out }}$ the fractional turnover rate (first-order loss of 5-HT through its removal from the extracellular space), and $I(C)$ the drug inhibitory function on the reuptake transporter of serotonin. The action 
Case Study 19

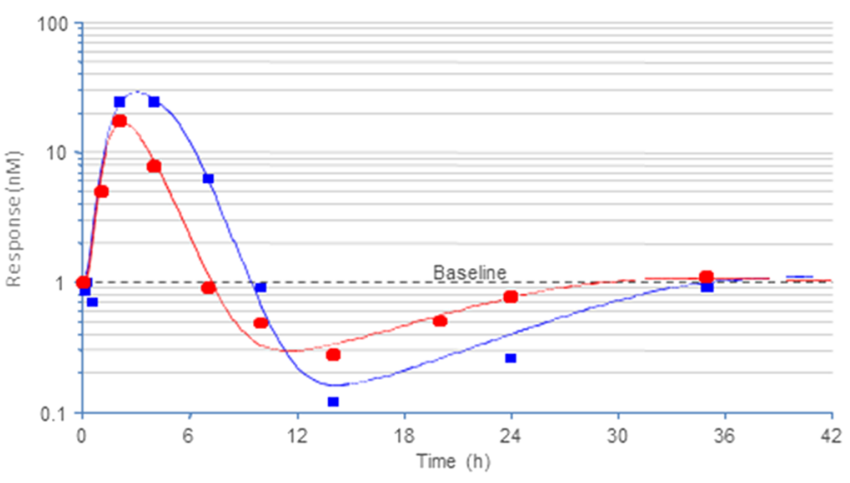

Case Study 20

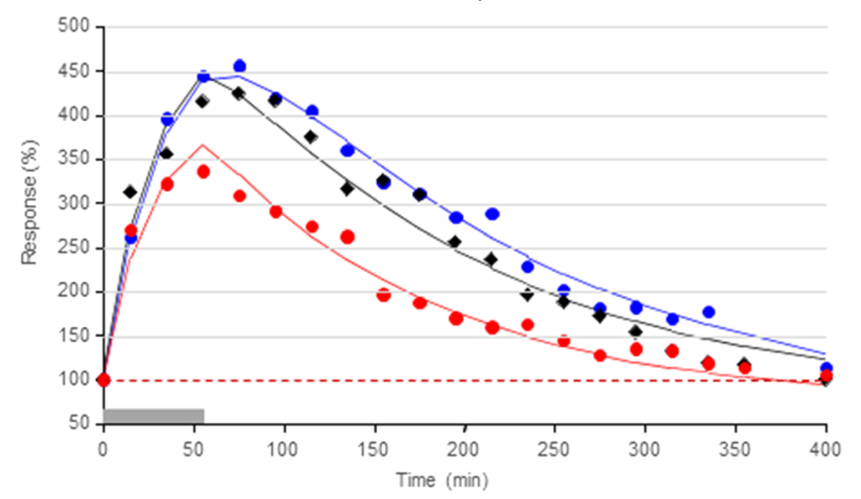

Fig. 12. Case study 19: semilogarithmic plot of experimental (symbols) and model predicted (lines) fold mRNA time data at two doses. Note the decline of mRNA below the predose baseline level (rebound) at both doses (high dose, blue squares; low dose, red dots). Also note the logarithmic response scale to more clearly show the relative change (increase or decrease) from the baseline (we used an adapted dataset with an alternative model than previously applied). Case study 20: observed (filled symbols) response vs. time from the three dose groups of rats after administration of 2.5 (red circles), 5 (black diamonds), and 10 (blue squares) $\mathrm{mg} / \mathrm{kg}$ of escitalopram as an intravenous infusion over $60 \mathrm{~min}$ (14). The inhibitory action of the loss of response $I(C)$ designates blockade of the serotonin reuptake transporters, resulting in elevated levels of serotonin, $R(27-31)$

of $M\left(M_{1}\right.$ and $M_{2}$; the receptor-mediated negative autoregulatory feedback) on $R$ was assumed to occur via inhibition of production of the response $R$ (level of 5-HT), which is attractive from a mechanistic and as well a neuronal resource management point of view. The turnover rate $k_{\text {in }}$ of $R$ was parameterized with the baseline value $R_{0}$ and fractional turnover rate $k_{\text {out }}$ as

$$
\left\{\begin{array}{l}
R_{0}=\sqrt{\frac{k_{\text {in }}}{k_{\text {out }}}} \\
k_{\text {in }}=R_{0}^{2} \cdot k_{\text {out }}
\end{array}\right.
$$

By setting Eq. 55 equal to zero (0) at baseline and rearranging the terms, $M_{01}$ and $M_{02}$ (baseline values of moderators $M_{1}$ and $M_{2}$ ) are equal to $R_{0}$ at baseline. The $k_{\mathrm{tol}}$ parameter is the first-order rate constant of the negative feedback mechanism, incorporating all of the aforementioned acutely "buffering" processes. At equilibrium, $M$ is equal to $R$, reflecting the balance between autoregulatory modulation and inhibition of reuptake to decrease and increase, respectively, the extracellular levels of 5-HT. According to the model, test compound concentrations $C$ act by blocking the reuptake of serotonin according to Eq. 57.

$I(C)=1-\frac{I_{\max } \cdot C^{n}}{\mathrm{IC}_{50}^{n}+C^{n}}$

where $I_{\max }$ is the efficacy (maximum drug-induced inhibition of the loss of response), $\mathrm{IC}_{50}$ the potency (plasma concentration reducing the maximum inhibition of loss of response by $50 \%$ ), and $n$ the sigmoidicity factor (steepness of the equilibrium concentration-response relationship at $\left.\mathrm{IC}_{50}\right)$. In the absence $\left(R_{0}, M_{0}\right)$ and presence $\left(R_{\mathrm{ss}}\right.$, $M_{s S}$ ) of the test compound, the baseline values of $R$ and $M$ become

$$
\left\{\begin{array}{l}
R_{0}=M_{01}=M_{02}=\sqrt{\frac{k_{\mathrm{in}}}{k_{\mathrm{out}}}} \\
R_{\mathrm{ss}}=M_{\mathrm{ss}}=\sqrt{\frac{k_{\mathrm{in}}}{k_{\mathrm{out}}} \cdot \frac{1}{I(C)}}=R_{0} \cdot \sqrt{\frac{1}{I(C)}}
\end{array}\right.
$$

The key patterns of this dataset are

- A predose baseline which remained constant throughout the experiment (data not shown)

- A rapid increase in the pharmacodynamic response

- A dose-dependent peak shift with response saturation at the two highest doses

- A parallel decline at all dose levels to predosing baseline when infusion is stopped

The peak shift indicates a nonlinear inhibitory function acting on the reuptake transport of 5-HT from the synaptic cleft back into the presynaptic storage compartment. The almost superimposable response-time courses of the intermediate and highest dose suggest the presence of functional counterregulatory adaptation. This is further highlighted by the concentration-response relationships for the three doses. Indeed, there is ample support for this kind of (acute) buffering processes from pharmacological studies. For example, marked increases in 5-HT beyond those induced by SSRI alone can be elicited by coadministration of agents blocking 5-HT $\mathrm{T}_{1 \mathrm{~A}}$ and 5$H T_{1 B}$ autoreceptors to attenuate the action of negative feedback mechanisms (e.g., 29,30).

\section{DISCUSSION}

Experimental protocol/study design may depend on implicit laboratory "traditions" as well as on the background education and scientific fostering of the investigator (pharmacologists vs. pharmacokineticists). However, we believe that there are some universal similarities with regard to how the actual outcome of an experiment is approached. Thus, 
from our personal experience, when looking at a responsetime course, the initial—subconsciously habitual—focus of a pharmacologist is to get a grip of what is conveyed by the overall shape of the curve: the development and change over time of exposure and a biomarker response (pharmacodynamic effect) following drug challenge. Interpretations based on this first glance will naturally have to take into account the biological system studied (the mechanism(s) of action), doses used (dose, rate, and route of administration), the very nature of the biomarker readout in question, its maximum efficacy, and any potential confounders to its expression (c.f. Table I). From a pharmacologist's point of view, the first look thus informs about

- Whether there is a delay in the onset of response, and if so, to what extent does it involve

- Route of administration (e.g., po vs. iv)

- Distributional factors (e.g., transport across barriers, access to biophase matrix)

- Biological system aspects (e.g., target matrix/ compartment and transduction vs. pharmacodynamic response variable studied, counterregulatory adjustments, cascading)

- Methodology (e.g., time resolution, sampling technique(s), other confounders)

- The intensity of the response and time to peak relative to exposure/doses

- The exposure-response relationship and saturation, assuming that more than one dose level is studied
- The duration and decay of the response relative to exposure

- Presence of pharmacodynamic rebound upon treatment cessation/drug washout

All of the above observations help in creating a preliminary pharmacological view on how exposure and biomarker variables may interact to generate the pattern observed. This information is then utilized to create hypotheses that may be formulated in mathematical model terms, and subsequently analyze how well the model fits the data observed.

From a pharmacokineticist's/modeler's point of view, pattern recognition is a pivotal aspect of the exploratory analysis process before modeling of data. Therefore, a careful strategy is essential for dissecting the patterns revealed by concentration-time, response-time, and concentrationresponse relations. This encompasses a set of points that specifically addresses baseline behavior, number of phases in data including convex or concave bending, time lags, peak shifts, shape of onset of action, intensity level, saturation, route-dependent response-time courses, duration of response, shape of decline of response, functional adaptation/buffering, synergistic effects, etc. (c.f. Table I). Signature profiles and proposed models are summarized in Figs. 3 and 4 in the "INTRODUCTION" section.

From our personal experience, many pharmacologists center their attention on absolute pharmacodynamic readouts, any associated issues, and mechanism-of-action resolution. Less priority is therefore put into the formulation and

Table I. Some Typical Features in Response-Time Data Interpreted by Pharmacologists and Modelers

\begin{tabular}{|c|c|c|}
\hline Features in data & Pharmacologist's view & Modeler's view \\
\hline Baseline & $\begin{array}{l}\text { Is the baseline response sufficiently defined and validated? If } \\
\text { not, what can be done to control for variation, drift, } \\
\text { confounders, and to maximize the pharmacodynamic } \\
\text { response window? }\end{array}$ & $\begin{array}{l}\text { Constant, oscillating, handling sensitive. What model } \\
\text { captures features of rest/sleep, handling, and disease } \\
\text { progression? }\end{array}$ \\
\hline Onset of action & $\begin{array}{l}\text { If delayed, to what extent are administration route-, drug } \\
\text { formulation-, PK-, and/or PD response-related (or } \\
\text { methodology) factors involved? }\end{array}$ & $\begin{array}{c}\text { Delayed or proceeds } C_{\max } \text {, initial rate of rise, steepness. } \\
\text { How can this be formalized in terms of equations? }\end{array}$ \\
\hline Intensity & $\begin{array}{l}\text { How does the response observed compare to what is expected } \\
\text { based on the biology of the system, including empirical } \\
\text { findings with other agents targeting the system? What is the } \\
\text { relation to dose/exposure? Has maximum efficacy been } \\
\text { reached, and to what extent does the maximum efficacy } \\
\text { reflect desired target action } v s \text {. nonspecific effects? }\end{array}$ & $\begin{array}{l}\text { Delayed or proceeds } C_{\max } \text {, high, low, dose-proportional, } \\
\text { saturated, waning, synergistic. Is there a physiological } \\
\text { limit or is it drug-dependent? }\end{array}$ \\
\hline Duration & $\begin{array}{l}\text { How does the duration relate to drug properties, underlying } \\
\text { physiology/biology } v s \text {. target characteristics and level of } \\
\text { drug exposure? What is the } t_{1 / 2} \text { of the PD response? }\end{array}$ & $\begin{array}{l}\text { Dose-proportional, offset, decline, steepness, regimen } \\
\text { dependent, monotonic decline, rebound. Is it caused by } \\
\text { drug or system properties? }\end{array}$ \\
\hline Acute findings & $\begin{array}{l}\text { To what degree does recruitment of counterregulatory } \\
\text { mechanisms limit expression of the acute PD response? If } \\
\text { present, how may such mechanisms be circumvented? }\end{array}$ & $\begin{array}{l}\text { Can data be used for prediction of chronic dosing? } \\
\text { Tolerance, adaptation, synergy }\end{array}$ \\
\hline Chronic findings & $\begin{array}{l}\text { Is the PD response size a/o profile altered upon chronic vs. } \\
\text { acute dosing? If so, what biology (tolerance, adaptation) vs. } \\
\text { PK-related factors may be involved? }\end{array}$ & $\begin{array}{l}\text { Can data be used for prediction of a specific response } \\
\text { upon chronic dosing? Tolerance, adaptation, synergy }\end{array}$ \\
\hline Rebound & $\begin{array}{l}\text { Is there an over- or undershoot in the PD action upon drug } \\
\text { withdrawal? What may be the cause of this, and can it be } \\
\text { avoided by altering drug, formulation, a/o treatment } \\
\text { scheduling properties? }\end{array}$ & $\begin{array}{l}\text { What is the area under the rebound vis-á-vis area under } \\
\text { response? Is there a dampened system? Can modeling } \\
\text { teach us something about avoiding the rebound effect? }\end{array}$ \\
\hline $\begin{array}{l}\text { Utilization of } \\
\text { information }\end{array}$ & $\begin{array}{l}\text { Creating a preliminary qualitative view on how drug } \\
\text { properties, exposure, and biological system variables may } \\
\text { interact to generate the pattern observed. }\end{array}$ & $\begin{array}{l}\text { Creating the input to model-based (quantitative) assess } \\
\text { ment of data. }\end{array}$ \\
\hline
\end{tabular}


expression of kinetic/dynamic relations in mathematical (quantitative) terms like in the equations in this paper, as modeling may appear cumbersome and less straightforward as an approach to the analysis of experimental data. We however believe that an even cursory conceptualization of the kinetic/dynamic outcome of any given experiment will help in avoiding throwing away valuable information, to the benefit of the compound and follow-up study design optimization.

The following list describes key features in a responsetime dataset from a modeling point of view:

- Baseline (stable, well-defined, or variable; oscillating or monotonically drifting; or zero/lack of baseline)

- Onset of action (delayed and/or later than $C_{\max }$, rise, steepness)

- Intensity (later than $C_{\max }$, high, low, dose-proportional, saturated, waning, synergistic)

- Duration (dose-proportional, offset, decline, steepness, regimen-dependent, monotonic decline, rebound)

- Repeated administration (tolerance, adaptation, synergy, disease progression)

A modeler's (pharmacokineticist's) view may focus primarily on the statistical aspects and less on the biological perspective. The generation of models may therefore become more empirical and numerical than mechanistic. However, below, we attempt to dissect and "translate" the components of the modeling equations into biological reasoning and underlying mechanistic explanations, in the hope to make the procedure more accessible and transparent. To generate a theoretical model, we first need to take into account likely processes and mechanisms defining baseline behavior and key factors influencing the chosen pharmacodynamic readout across the time frame of the experiment. Whenever possible, it is recommendable to include richer datasets, particularly information about the nature of baseline behavior (variability). If robust baseline information is available, such data should ideally be part of the model. It is also suggested that analyses based on baseline (or vehicle)-subtracted relative (\%) effects should be avoided (32).

\section{Standard, Nondrifting, Baseline Situation}

Some central themes for the data patterns and some of their determinants are summarized below. The change in response $R$ over time can be expressed in terms of a differential equation $\mathrm{d} R / \mathrm{d} t$ (Eq. 59 top) starting with the situation at baseline (i.e., in the absence of pharmacological or physiological challenge, Eq. 59 bottom). A baseline response is a consequence of turnover rate $k_{\text {in }}$ and loss $k_{\text {out }}$.

$$
\left\{\begin{array}{l}
\frac{\mathrm{d} R}{\mathrm{~d} t}=k_{\mathrm{out}} \cdot R_{0}-k_{\mathrm{out}} \cdot R \\
\text { Baseline }=R_{0}=\frac{k_{\mathrm{in}}}{k_{\mathrm{out}}}
\end{array}\right.
$$

For example, in the case where the buildup of a defined response via stimulation of $k_{\text {in }}$ is the major determinant, Eq. 59 becomes

$$
\left\{\begin{array}{l}
\frac{d R}{d t}=k_{\text {out }} \cdot R_{0} \cdot S(C)-k_{\text {out }} \cdot R \\
R_{\text {ss }}=\frac{k_{\text {in }}}{k_{\text {out }}} \cdot S(C)=R_{0} \cdot S(C)
\end{array}\right.
$$

\section{Baseline Equal to Zero}

When the baseline is essentially zero, $R$ can be modeled as in Eq. 61

$$
\left\{\begin{array}{l}
\frac{\mathrm{d} R}{\mathrm{~d} t}=S(C)-k_{\text {out }} \cdot R \\
\text { Baseline }=0 \\
R_{\mathrm{ss}}=\frac{S(C)}{k_{\text {out }}}
\end{array}\right.
$$

In this particular case, the steady-state response $R_{\mathrm{ss}}$ is then only determined by the drug "mechanism" function and $k_{\text {out }}$. This kind of situation could for example apply to studies measuring locomotor activity in habituated or monoaminedepleted rodents, which remain essentially immobile until challenged with agents able to promote (directly or indirectly) dopaminergic neurotransmission (e.g., van Rossum and van Koppen (13)). Once the stimulant drug is then eliminated from the body, the locomotor response again returns to its pretreatment "zero" baseline-as illustrated in case study 5 above.

\section{Drifting or Oscillating Baseline}

The baseline response sometimes drifts down- or upward over time. This naturally affects the modeling equations, such that the ratio between $k_{\text {in }}$ and $k_{\text {out }}$ at baseline will be smaller or greater than unity, respectively. Thus, for example, in case study 9 (Fig. 8 ), the rate of tumor growth $\left(k_{\text {in }}\right)$ clearly exceeds that of apoptosis $\left(k_{\text {out }}\right)$, resulting in a vehicle curve approaching an exponential increase of the response (tumor volume). Drug treatment in this situation led to rightward shifts of the curve with rising doses and at the highest dose even to a period of "reversed" growth-suppression/tumor shrinkage-until following drug washout when tumor volume expansion re-emerged. The different curve shapes in this example therefore also hints about the relative impact of the drug on production (= growth) vs. loss (= "kill") mechanisms at different levels of exposure.

The baseline response may also display an oscillating behavior (e.g., Fig. 10, case study 15) which if so can be represented by means of a cosine input function.

$$
\left\{\begin{array}{l}
R_{0}=\frac{k_{\text {in }}(t)}{k_{\text {out }}} \\
R_{\mathrm{ss}}=\frac{k_{\text {in }}(t)}{k_{\text {out }}} \cdot S(C)
\end{array}\right.
$$

This type of baseline pattern is typically observed in the context of endogenous hormonal responses, which may 
display regular infradian, circadian, or ultradian cycles. It may also be as in case study 14 (Fig. 9), where an oscillating drug "mechanism" function in itself is governed by an oscillating endogenous agonist "driver." In either of these situations, it is evident that awareness of the underlying physiology is a prerequisite for the formulation of a suitable modeling approach.

Needless to say, a deeper insight through visual pattern recognition and the identification of a matching model may-as illustrated i.a. by the above examples-benefits the optimization of drug as well as treatment protocol design. We are convinced that the reader will have several examples from their own experience attesting to this view.

\section{Onset of Action}

The onset of action may manifest itself as a concave buildup of the response $R$ and is mimicked by a series of transit compartments prior to the compartment that represents the measured pharmacological response $R_{i}$. As an example, the activation of the target receptor $\left(R_{\text {receptor }}\right)$ may require a series of downstream (transduction/transcription/ translation/signaling, etc.) cascade events $\left(R_{1}-R_{i}\right)$ with varying time frames and efficiency before the final pharmacodynamic readout response $R$ can be observed. This process may thus be expressed as in the modeling Eq. 66 below-attempting to capture the transition phases in between the very ligand-receptor binding at the prevailing level of exposure $(S(C))$, and the final degree of response $R$, relative to the baseline pharmacodynamic response $R_{0}$.

$$
\begin{aligned}
& \frac{\mathrm{d} R_{\text {receptor }}}{\mathrm{d} t}=k_{\text {out }} \cdot\left(R_{0} \cdot S(C)-R_{\text {receptor }}\right) \\
& \frac{\mathrm{d} R_{1}}{\mathrm{~d} t}=k_{\text {out }} \cdot\left(R_{\text {receptor }}-R_{1}\right) \\
& \frac{\ddot{\mathrm{d}} R_{i-1}}{\mathrm{~d} t}=k_{\text {out }} \cdot\left(R_{i-2}-R_{i-1}\right) \\
& \frac{\mathrm{d} R_{i}}{\mathrm{~d} t}=k_{\text {out }} \cdot\left(R_{i-1}-R_{i}\right)
\end{aligned}
$$

This type of function not only accounts for delays in the onset but also in the duration of response. Amplification of a signal within the series of transit steps is taken care of by Eq. 64, wherein $R_{i-1}^{\gamma}$ represents the response at the defined transit step, with the index $\gamma$ designating the level of amplification

$\frac{\mathrm{d} R_{i}}{\mathrm{~d} t}=k_{\mathrm{out}} \cdot\left(R_{i-1}^{\gamma}-R_{i}\right)$

A peak shift in the maximum response with increasing doses suggests a nonlinear drug "mechanism" function, provided there is no peak shift in plasma concentration. If the intensity of response displays saturation at higher doses, it may be a consequence of a nonlinear drug "mechanism" function or a physiological limit. To discriminate between these two factors, independent types of provocations of the response-time course may be needed to dissect the origin of the observation.

$$
\left\{\begin{array}{l}
I(C)=1-\frac{I_{\max } \cdot C^{n}}{\mathrm{IC}_{50}^{n}+C^{n}} \\
S(C)=1+\frac{S_{\max } \cdot C^{n}}{\mathrm{SC}_{50}^{n}+C^{n}}
\end{array}\right.
$$

An alternative drug "mechanism" function is with exponential growth of inhibition.

$$
\left\{\begin{array}{l}
I(C)=1-(a \cdot C)^{n} \\
S(C)=1+(a \cdot C)^{n}
\end{array}\right.
$$

If maximum response occurs at the same time point independent of dose, it may be prudent to consider a linear drug "mechanism" function acting on the turnover rate

$$
\left\{\begin{array}{l}
I(C)=1-a \cdot C \\
S(C)=1+a \cdot C
\end{array}\right.
$$

If this observation is coupled to an observed saturation, some other model (e.g., link) structure than a turnover model has to be considered. Drug action on factors responsible for the loss of response $\left(k_{\text {out }} \cdot I(C)\right.$ or $\left.k_{\text {out }} \cdot S(C)\right)$ will always cause a leftward or rightward peak shift in response with a change in dose since half-life of response changes. Dose-normalized areas under the response-time courses plotted vs. dose will reveal whether the drug "mechanism" function behaves linearly (horizontal line) or nonlinearly (increasing or decreasing with dose).

\section{Duration of Response}

The duration of the response relative to the drug exposure is a very important piece of information for the analysis and modeling of kinetic/dynamic data. There may be several potential reasons for the disconnect between drug exposure and pharmacodynamic response. Such as, indirect action on factors determining the response (e.g., Fig. 5, case study 1 ; Fig. 9 , case study 14 ), the pharmacodynamic response may attenuate despite sustained drug levels (e.g., Fig. 10, case study 16), and/or there may be a rebound response following elimination of the drug from the system (e.g., Fig. 7, case study 6; Fig. 10, case study 18). All of the aforementioned examples require insight into the underlying cause(s), both in terms of pharmacokinetic-related and biological system/ pharmacodynamic-related factors, in order for a suitable modeling approach to be generated.

\section{CONCLUDING REMARKS}

The objective of this communication has been to focus on the power of what may be initially captured by visual inspection of "shapes" or "patterns" of response-time profiles. We have then tried to decompose these "shapes" and proposed what determines the rise, intensity, and decline of response. This is then described in terms of mathematical expressions of turnover (indirect response) models. This 
approach may serve as a road map to pattern recognition of response-time data. It is our hope that the descriptions will facilitate for the presumptive reader the analysis and interpretation of kinetic/dynamic data. The pattern recognition approach to response curve shapes will assist the selection of suitable modeling approaches to the actual experiment, and to superior design of any follow-up studies. Hence, by optimally utilizing information displayed openly (or sometimes in disguise...) in the study data, an improved understanding of target biology as well as of the various components determining drug action will be accomplished.

As evident from many of the case studies in this tutorial, the pharmacodynamic response curves can be more or less disconnected from plasma exposure time courses. In this respect, it seems that time delays are particularly common, likely because biological system processes typically involve transduction cascades, "buffering" mechanisms, organ/tissue interplay processes, etc. In addition, some compounds interact with their targets in a more or less irreversible fashion. By applying a pattern recognition approach, detailed knowledge of drug compound properties, and thorough insight into the underlying biology of the response under study, a superior modeling of the experimental readout can be attained. This is clearly different from the routine, empirical approach sometimes taken, but that should always be avoided ( $c f$. "They were so intent making everything numerical, that they frequently missed seeing what was there to be seen."; (33)). Finally, to this end, we cannot emphasize enough the importance of tight collaboration between pharmacology and DMPK-oriented scientists in the interpretation of experimental readouts.

\section{ACKNOWLEDGMENTS}

Data of case study 1 were originally generated and analyzed by Dr. C. Dagenais at AstraZeneca, Wilmington, DE, and were then generously shared during numerous workshops within and outside AZ. Data of case study 9 were generated at AstraZeneca, Waltham, MA, and then adapted to suit the purpose of pattern recognition. The preliminary data of case study 19 were generously shared by D. Hovdal, AstraZeneca AB, Mölndal, Sweden. An alternative model to the originally used was then applied.

\section{REFERENCES}

1. Duan JZ. Drug-drug interaction pattern recognition. Drugs R D. 2010;10:9-24.

2. Gabrielsson J, Meibohm B, Weiner D. Pattern recognition in pharmacokinetic analysis. AAPS J. 2015. doi:10.1208/s12248-0159817-6.

3. Dayneka NL, Garg V, Jusko WJ. Comparison of four basic models of indirect pharmacodynamic responses. J Pharmacokinet Biopharm. 1993;21:457-78.

4. Gabrielsson J, Weiner D. Pharmacokinetic and pharmacodynamic data analysis: concepts and applications, 1st-4th edition, Swedish Pharmaceutical Press, Stockholm (1994-2010).

5. Jusko WJ, Ko HC. Physiologic indirect response models characterize diverse types of pharmacodynamic effects. Clin Pharmacol Ther. 1994;56:406-19.

6. Paalzow LK, Edlund PO. Multiple receptor responses: a new concept to describe the relationship between pharmacological effects and pharmacokinetics of a drug: studies on clonidine in the rat and cat. J Pharmacokin Biopharm. 1979;7:495-510.
7. Jumbe NL, Xin Y, Leipold DD, Crocker L, Dugger D, Mai E, et al. Modeling the efficacy of trastuzumab-DM1, an antibody drug conjugate, in mice. J Pharmacokinet Pharmacodyn. 2010;37:221-42. doi:10.1007/s10928-010-9156-2.

8. Zhi J, Nightingale $\mathrm{CH}$, Quintiliani R. Microbial pharmacodynamics of piperacillin in neutropenic mice of systematic infection due to Pseudomonas aeruginosa. J Pharmacokin Biopharm. 1988;16:355-75.

9. Chakraborty A, Krzyzanski W, Jusko WJ. Mathematical modeling of circadian cortisol concentrations using indirect response models: comparison of several methods. J Pharmacokinet Biopharm. 1999;27:23-43.

10. Ramakrishnan R, DuBois DC, Almon RR, Pyszczynski NA, Jusko WJ. Fifth-generation model for corticosteroid pharmacodynamics: application to steady-state receptor down-regulation and enzyme induction patterns during seven-day continuous infusion of methylprednisolone in rats. J Pharmacokinet Pharmacodyn. 2002;29:1-24.

11. Jusko WJ. Guidelines for collection and analysis of pharmacokinetic data. In: Burton ME, Shaw LM, Schentag JJ, Evans WE, editors. Applied pharmacokinetics: principles of therapeutic drug monitoring, 4th edition. Philadelphia: Lippincott Williams \& Wilkins; 2006.

12. Nagashima R, O'Reilly RA, Levy G. Kinetics of pharmacologic effects in man: the anticoagulant action of warfarin. Clin Pharmacol Ther. 1969;10:22-35.

13. van Rossum JM, van Koppen AT. Kinetics of psycho-motor stimulus drug action. Eur J Pharmacol. 1968;2:405-8.

14. Gabrielsson J, Peletier LA. Dose-response-time data analysis involving nonlinear dynamics, feedback and delay. Eur J Pharm Sci. 2014;59:36-48. doi:10.1016/j.ejps.2014.04.007.

15. Goodman \& Gilman's the pharmacological basis of therapeutics, 12th ed. In: Brunton LL, Chabner BA, Knollmann BC, editors. MacGraw Hill Education; 2012.

16. Krzyzanski W, Ramakrishnan R, Jusko WJ. Basic pharmacodynamic models for agents that alter production of natural cells. $\mathrm{J}$ Pharmacokinet Biopharm. 1999;27:467-89.

17. Fong TM, Heymsfield SB. Cannabinoid-1 receptor inverse agonists: current understanding of mechanism of action and unanswered questions. Int J Obes (Lond). 2009;33:947-55.

18. Siemers ER, Dean RA, Friedrich S, Ferguson-Sells L, Gonzales C, Farlow MR, et al. Safety, tolerability, and effects on plasma and cerebrospinal fluid amyloid-beta after inhibition of gammasecretase. Clin Neuropharmacol. 2007;30:317-25.

19. Gabrielsson J, Peletier LA. Mixture dynamics: dual action of inhibition and stimulation. Eur J Pharm Sci. 2013;50:215-26.

20. Peletier LA, Gabrielsson J, den Haag J. A dynamical systems analysis of the indirect response model with special emphasis on time to peak response. J PharmacokinPharmacodyn. 2005;32:607-54.

21. Gatto GJ, Bohme GA, Caldwell WS, Letchworth SR, Traina VM, Obinu MC, et al. TC-1734: an orally active neuronal nicotinic acetylcholine receptor modulator with antidepressant, neuroprotective and long-lasting cognitive effects. CNS Drug Rev. 2004:10:147-66.

22. Smolen VF. Quantitative determination of drug bioavailability and biokinetic behavior from pharmacological data for ophthalmic and oral administration of a mydriatic drug. J Pharm Sci. 1971;60:354-63.

23. Tao R, Hjorth $\mathrm{S}$. Differences in the in vitro and in vivo 5hydroxytryptamine extraction performance among three common microdialysis membranes. J Neurochem. 1992;59:1778-85.

24. Ekstrand $\mathrm{C}$, Ingvast-Larsson $\mathrm{C}$, Olsén L, Hedeland $\mathrm{M}$, Bondesson U, Gabrielsson J. A quantitative approach to analysing hydrocortisone response in the horse. J Vet Pharmacol Ther. 2015. doi:10.1111/jvp.12276.

25. Zannikos PN, Rohatagi S, Jensen BK. Pharmacokineticpharmacodynamic modeling of the antilipolytic effects of an adenosine receptor agonist in healthy volunteers. J Clin Pharmacol. 2001;41:61-9.

26. Isaksson C, Wallenius K, Peletier LA, Toresson H, Gabrielsson $\mathrm{J}$. Turnover modeling of non-esterified fatty acids in rats after multiple intravenous infusions of nicotinic acid. Dose-Response. 2009;7:247-69. 
27. Bundgaard C, Larsen F, Jørgensen M, Gabrielsson J. Mechanistic model of acute autoinhibitory feedback action after administration of SSRIs in rats: application to escitalopraminduced effects on brain serotonin levels. Eur J Pharm Sci. 2006;29:394-404.

28. Peletier LA, Gabrielsson J. A nonlinear feedback model capturing different patterns of tolerance and rebound. Eur J Pharm Sci. 2007;32:85-104.

29. Hjorth S. Serotonin $5 \mathrm{HT}_{1 \mathrm{~A}}$ autoreceptor blockade potentiates the ability of the 5-HT reuptake inhibitor citalopram to increase nerve terminal 5-HT output in vivo: a micro-dialysis study. J Neurochem. 1993;60:776-9.
30. Hjorth S, Auerbach SB. 5-HT $1 \mathrm{~A}$ autoreceptors and the mode of action of selective serotonin reuptake inhibitors (SSRI). Behav Brain Res. 1995;73:281-3.

31. Hjorth S, Bengtsson HJ, Kullberg A, Carlzon D, Peilot H, Auerbach SB. Serotonin autoreceptor function and antidepressant drug action. J Psychopharmacol. 2000;14:177-85.

32. Gabrielsson J, Hjorth S, Vogg B, Harlfinger S, Gutierrez PM, Peletier LA, et al. Modeling and design of challenge tests: inflammatory and metabolic biomarker study examples. Eur J Pharm Sci. 2015;67:144-59.

33. Keller FE. A feeling for the organism: the life and work of Barbara McClintock. New York: W. H. Freeman; 1983. 